\title{
IMPORTANCIA CULTURAL DE LA FLORA SILVESTRE DEL DISTRITO DE PAMPAROMÁS, ANCASH, PERÚ
}

\section{CULTURAL IMPORTANCE OF THE WILD FLORA OF THE DISTRICT OF PAMPAROMÁS, ANCASH, PERU}

\author{
Roxana Castañeda Sifuentes ${ }^{1,2,3}$ y Joaquina Albán Castillo ${ }^{1}$
}

\begin{abstract}
Resumen
El presente estudio etnobotánico se realizó en el distrito de Pamparomás. Los objetivos desarrollados estuvieron referidos en rescatar el conocimiento ancestral asociado a la flora silvestre y determinar las especies de mayor importancia cultural. La metodología aplicada en la investigación se basó en la ejecución de entrevistas informales y semiestructuradas a 202 informantes locales. Los datos de las entrevistas informales fueron obtenidos mediante preguntas abiertas durante las caminatas etnobotánicas. Las entrevistas semiestructuradas se realizaron con la ayuda de algunos instrumentos visuales (herbario portátil y panel fotográfico). Para estimar la importancia de las plantas silvestres se empleó el índice de Importancia Cultural (IC) propuesto por Tardío y Pardo-de-Santayana (2008). Se reportan un total de 237 especies útiles agrupadas en 189 géneros y 68 familias. La familia Asteraceae reportó el mayor número de especies seguida de Poaceae y Fabaceae. Asimismo, las especies fueron agrupadas en nueve categorías de uso, siendo la categoría medicinal la que presentó el mayor número de especies y la que registró los mayores reportes de uso. Las especies vegetales con mayor IC son Alnus acuminata (Ramrash, aliso), Iochroma umbellatum (Rukii, San Pablo, puya, quebrolla) y Polylepis racemosa (Quenua, quenual, quiñua), y son estas las especies más ligadas al acervo cultural de los pobladores pamparominos.
\end{abstract}

Palabras clave: Andes, conocimiento tradicional, flora silvestre, importancia cultural, plantas vasculares.

\begin{abstract}
This ethnobotanical study was conducted in the district of Pamparomás. The objectives of the study were centered upon the task of recovering ancestral knowledge associated with wild flora and identifying those species of greatest cultural importance. The methodology applied to the research was based upon the conducting of informal and semi-structured interviews to 202 local informants. Data from the informal interviews were obtained by means of open questions during ethnobotanical walks. The semi-structured interviews were conducted with the help of certain visual aids (portable herbarium and photographic panels). In order to determine the importance of wild plants, the Cultural Importance Index (CI) proposed by Tardío and Pardo-de-Santayana (2008) was employed. A total of 237 useful species were reported, grouped into 189 genera and 68 families. The highest number of species was found to belong to the Asteraceae family, followed by the Poaceae and Fabaceae families. Also, the species were grouped into nine categories of use, with the medicinal category containing the highest number of species and registering the greatest reported usage. The plant species with the highest CI were Alnus acuminata (ramrash, alder), Iochroma umbellatum (rukii, San Pablo, puya, quebrolla) and Polylepis racemosa (quenua, quenual, quiñua). It was these species which were found to be most closely associated with the cultural heritage of the people of Pamparomás.
\end{abstract}

Key words: Andes, traditional knowledge, wild flora, cultural importance, vascular plants.

\section{Introducción.}

Las plantas son de vital importancia para el hombre porque permiten satisfacer sus necesidades de supervivencia, ya sea como alimento, como ornamento, para producir calor, para abrigarse, para el cuidado de la salud, el arreglo personal, en la construcción, producción de tintes, entre otros (Albán, 1998; Levy \& Aguirre, 1999).

Los usos de las plantas están relacionados con las creencias y patrones de comportamiento de los seres humanos de acuerdo a su rol social, ello cobra importancia porque a partir de investigaciones que 
cuantifiquen el conocimiento tradicional asociado a la flora, se pueden identificar especies vegetales que merecen estudios más profundos, dándole validez y confiabilidad a los datos proporcionados por los informantes (Castañeda, 2011).

En los últimos 30 años, ha habido un creciente interés en mejorar el estilo de compilación tradicional de los estudios etnobotánicos mediante la incorporación de métodos de investigación cuantitativa en la colección de información, procesamiento e interpretación de los resultados. Los métodos cuantitativos proporcionan un enfoque adicional para conocer las plantas que son consideradas más importantes para el poblador, puesto que ayudan a jerarquizar a las plantas útiles, en base al conocimiento de uso que le otorga un grupo humano (Martínez, 1991).

Los métodos cuantitativos en etnobotánica, se agrupan en tres enfoques: Usos totales, Asignación subjetiva y Consenso de informantes; dentro del enfoque de Consenso de informantes la importancia relativa de cada uso es calculada directamente a partir del grado de consenso entre las respuestas de los informantes, esto permite realizar comparaciones estadísticas por lo que se considera relativamente objetiva (Castañeda, 2011); este enfoque ha sido motivo de numerosos estudios en los cuales se han planteado diferentes índices culturales para poder estimar la significancia de las plantas para determinados grupos humanos.

El índice de Importancia Cultural, propuesto por Tardío \& Pardo-de-Santayana (2008), sirve para identificar las plantas de mayor significancia cultural para una determinada comunidad en base a los reportes de uso por especie. Es así que en el presente trabajo se empleó este índice para estimar la Importancia Cultural de la flora silvestre del distrito de Pamparomás.

Pamparomás se encuentra ubicado en la Falda Occidental de la Cordillera Negra del Callejón de Huaylas, en el departamento de Ancash. Se ha elegido esta comunidad, por representar una población que a pesar de haber sufrido las consecuencias de las acciones colonizadoras (manifestadas por la usurpación de sus territorios, sus recursos, así como de la apropiación de sus fuerzas de trabajo) conservan aún su propia cultura (Moreno, 1966).

Estudios previos en la zona fueron realizados por Moreno (1966), quien muestra las riquezas culturales en el ámbito pamparomino, refiriendo que cada centro poblado posee su folklore propio que -en sus diversas manifestaciones- debemos tratar de conservar, cultivar y documentar con el objeto de servir como herencia del pasado, reafirmación del presente y fuente de inspiración del futuro. Moreno (1989) evidenció el panorama global del distrito enfatizando cada una de sus riquezas y actividades como parte de un programa de desarrollo integral. Albán (1998) recopiló la
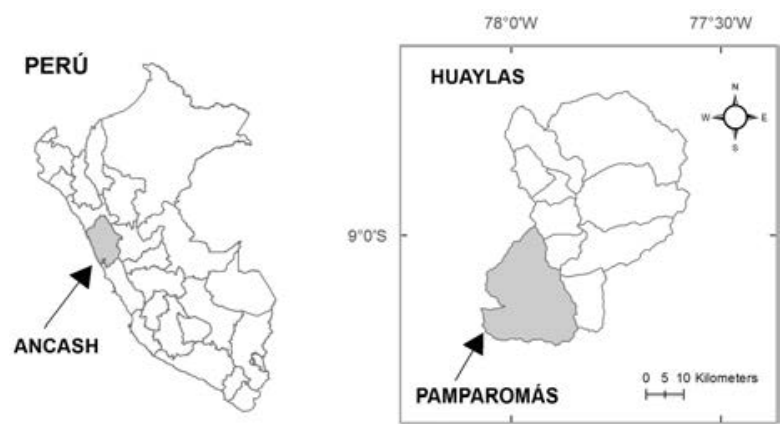

Figura 1. Mapa de ubicación del distrito de Pamparomás, Huaylas, Ancash.

información sobre los usos que los pobladores pamparominos atribuyen a sus recursos vegetales, teniendo en cuenta la distribución de estos en los diversos pisos de vegetación; adiciona, además, la sinonimia vernácula para cada una de las 747 especies descritas, según su concepción tradicional y en relación a sus usos. Alvarado (2007) realizó un estudio sobre las plantas medicinales de la Cordillera Negra, dándole un enfoque fitoquímico. Castañeda (2011) estimó el Valor Cultural de las plantas silvestres de Pamparomás, Marcó y Cajabamba Alta. Asimismo, Castañeda (2014) comparó tres índices de significancia cultural, tomando como base la información sobre los usos de las plantas silvestres del caserío de Pisha. También, Castañeda et al. (2014) realizaron el inventario de las plantas silvestres empleadas como alimento para animales en el caserío de Pisha. Finalmente, Melchor (2016) ejecutó un estudio etnobotánico sobre la familia Fabaceae de la cuenca del río Nepeña.

El presente estudio deriva de tres tesis etnobotánicas ejecutadas en el distrito de Pamparomás (Albán, 1998; Castañeda, 2011 y Castañeda, 2014), tiene por finalidad documentar la riqueza de la flora silvestre útil del distrito y determinar las especies vegetales más importantes para la vida del poblador pamparomino mediante el uso del índice de Importancia Cultural.

\section{Materiales y métodos.}

Área de estudio

La investigación se realizó en el distrito de Pamparomás, provincia de Huaylas, región Ancash. El ámbito de estudio se encuentra ubicado en la falda occidental de la Cordillera Negra y presenta una extensión, alrededor de $496.4 \mathrm{~km}^{2}$ (Albán, 1998). Se encuentra comprendido entre los 1300 y 5006 msnm y se ubica entre los $8^{\circ} 88^{\prime} 32^{\prime \prime}$ - 9 ${ }^{\circ} 15^{\prime} 06^{\prime \prime} \mathrm{S}$ y $77^{\circ} 50^{\prime} 29^{\prime \prime}$ 7803'24" W (Figura 1). Debido al amplio rango de altitudes, Pamparomás presenta climas cálidos cuyas temperaturas varían entre los $20^{\circ} \mathrm{C}$ y $27^{\circ} \mathrm{C}$ y climas templados a fríos con temperaturas entre los $0^{\circ} \mathrm{C} \mathrm{y}$ $10^{\circ} \mathrm{C}$ (Albán, 1998). Pamparomás es un distrito andino cuyas actividades económicas principales son la 
Tabla 1. Categorías de uso de la flora silvestre útil del distrito de Pamparomás, Ancash.

\begin{tabular}{ll}
\hline $\mathbf{N}^{\mathbf{0}}$ & Categoría de uso \\
\hline 1 & $\begin{array}{l}\text { Alimenticio (ALI): Especies consumidas directa o } \\
\text { indirectamente por el hombre. }\end{array}$
\end{tabular}

2 Alimento para animales (APA): Especies consumidas por animales domésticos y silvestres, vertebrados o invertebrados. Se incluyen las plantas forrajeras y melíferas.

3 Ambiental (AMB): Especies que proporcionan bienes y servicios al hombre, desempeñando, además varias funciones ecológicas. Incluye plantas empleadas en la agroforestería, como ornamentales, árboles para sombra, como cercos vivos, entre otros.

4 Combustible (COM): Especies utilizadas para la elaboración de leña o carbón.

5 Etnoveterinario (ETN): Especies utilizadas como medicinales para los animales. Se incluyen las plantas abortivas para animales.

6 Materiales (MAT): Especies utilizadas como fuentes de materia prima utilizadas en los distintos aspectos de subsistencia del hombre y su ambiente.

7 Medicinal (MED): Especies vinculadas al tratamiento de las dolencias y percepciones patológicas sensibles al hombre, así como los síndromes (conjunto de síntomas que caracterizan una enfermedad). Incluye especies empleadas para curar enfermedades culturales como el "susto", "mal de aire” y "mal de ojo”. También se incluyen las plantas abortivas para humanos.

8 Social (SOC): Especies utilizadas con propósitos culturales diversos. Esta categoría incluye especies vinculadas al sistema de creencias de la comunidad, y que en su conjunto sustentan la cosmovisión del grupo humano que se estudia. Incluye a las plantas utilizadas con fines mágicos, religiosos y ritualistas.

9 Tóxica (TOX): Especies consideradas venenosas para el hombre $\mathrm{y} / \mathrm{o}$ animales de manera accidental o intencional. Incluye especies empleadas como herbicidas e insecticidas.

agricultura y la ganadería. Los pobladores hablan el idioma Quechua.

Recolección del material botánico

La recolección del material botánico fue realizado con la autorización de investigación y colecta de muestras de herbario de flora silvestre, otorgada por el Ministerio de Agricultura a través de la Resolución Directoral $\mathrm{N}^{\circ}$ 030-2012-AG-DGFFS-DGEFFS. Las muestras botánicas fueron recolectadas durante el periodo comprendido entre los años 1990 y 2014 realizando dos o tres viajes anuales de una duración de 15 a 25 días. El trabajo de campo fue realizado por las autoras como parte de tres tesis: Albán (1998), Castañeda (2011) y Castañeda (2014).

Los ejemplares botánicos fueron procesados según el método convencional (Cerrate, 1964). La determinación taxonómica fue realizada en el Departamento de Etnobotánica y Botánica Económica del Museo de Historia Natural de la Universidad Nacional Mayor de San Marcos. Se realizaron consultas en el Herbario San Marcos (USM) y se recurrió a diversos especialistas en los diferentes taxa.

Las especies han sido clasificadas según Angiosperm Phylogeny Group (APG IV, 2016). La nomenclatura fue consultada en la base de datos Tropicos (2016) del Missouri Botanical Garden. Se consultó publicaciones especializadas que respaldan la nomenclatura empleada en el presente trabajo (Lodé \& Pino, 2008; Chemisquy et al., 2010). Para la redacción de los nombres comunes en idioma Quechua se consultó el libro de gramática del quechua ancashino de Julca (2009). Los ejemplares obtenidos fueron depositados en la colección del Herbario San Marcos (USM).

Registro de información etnobotánica

Se solicitó el consentimiento informado previo a los pobladores de Pamparomás, explicando en una asamblea comunal los objetivos y alcances de la presente investigación. Para la toma de datos etnobotánicos se emplearon entrevistas informales y semiestructuradas (Alexiades, 1996). Se realizaron 202 entrevistas a informantes locales. Los datos de las entrevistas informales fueron obtenidos mediante preguntas abiertas, anotando en una libreta de campo la información proporcionada por los pobladores durante las caminatas etnobotánicas. Las entrevistas se realizaron con la ayuda de un herbario portátil, un panel fotográfico en computadora portátil y fichas modificadas a partir de Albán (1985) y Castañeda (2014) (Apéndice 1).

Determinación de la Importancia Cultural

Para identificar las especies vegetales de mayor significancia cultural, se empleó el índice de Importancia Cultural (IC) propuesto Tardío \& Pardode-Santayana (2008) que se basa en los reportes de uso por especie. El Reporte de Uso (RU) es el evento en el cual el informante i menciona el uso de la especies e en la categoría de uso u (Kufer et al., 2005).

$$
\text { ICe }=\sum_{\mathrm{u}=\mathrm{u} 1}^{\mathrm{uNC}} \sum_{\mathrm{i}=\mathrm{i} 1}^{\mathrm{iN}} \text { RUuie/N }
$$

Dónde:

ICe $=$ Importancia cultural de la especie e.

RUuie $=$ Reportes de uso de la especie e.

$\mathrm{N} \quad=$ Número de informantes considerados en el estudio.

El índice IC puede ser considerado como una simplificación del Valor Cultural planteado por Reyes-García et al. (2006) siendo el tercer factor del índice de Valor Cultural; aunque también puede ser visto como una redefinición del Valor de Uso de Phillips \& Gentry (1993).

La Importancia Cultural varía de 0 (cuando nadie menciona el uso de ninguna planta) al número de categorías de uso (en el hipotético caso de que todos los informantes mencionen que todas las especies 
sirvan para todas las categorías de uso que se consideran en el estudio). Por lo tanto, en esta investigación en particular el índice de IC varía de 0 a 9.

\section{Categorías de uso}

Las categorías de uso empleadas en el presente trabajo han sido adaptadas de Paniagua et al. (2010), Albán (2013) y Gruca et al. (2014) (Tabla 1).

\section{Resultados.}

Se registraron 237 especies, 189 géneros y 68 familias de plantas vasculares silvestres, agrupadas en nueve categorías de uso. En el Apéndice 2 se muestra la lista de todas las especies reportadas en orden alfabético de familias, indicando para cada una el número de reportes de uso por categorías de uso, así como los reportes de usos acumulados y la Importancia Cultural de cada planta.

La familia con mayor riqueza de especies es Asteraceae con 44 especies (18\%), seguida por Poaceae con 24 especies (10\%), Fabaceae con 16 especies (7\%) y Solanaceae con 15 especies (6\%) (Figura 2).

La categoría medicinal presenta el mayor número de reportes de uso (1587), seguida de las categorías alimento para animales (786) y combustible (595) (Figura 3).

De igual modo la categoría medicinal presenta la mayor riqueza de especies (140), seguida de las categorías alimento para animales (123) y combustible (62) (Figura 4).

En la Tabla 2 se muestran las 10 especies vegetales de mayor Importancia Cultural agrupadas por orden de mérito, indicando para cada una el número de reportes de uso por categorías de uso, así como los reportes de usos acumulados (RU) y la Importancia Cultural (IC) de cada planta.

En la Figura 5 se muestra el número de reportes de usos para las categorías sobresalientes de las cinco

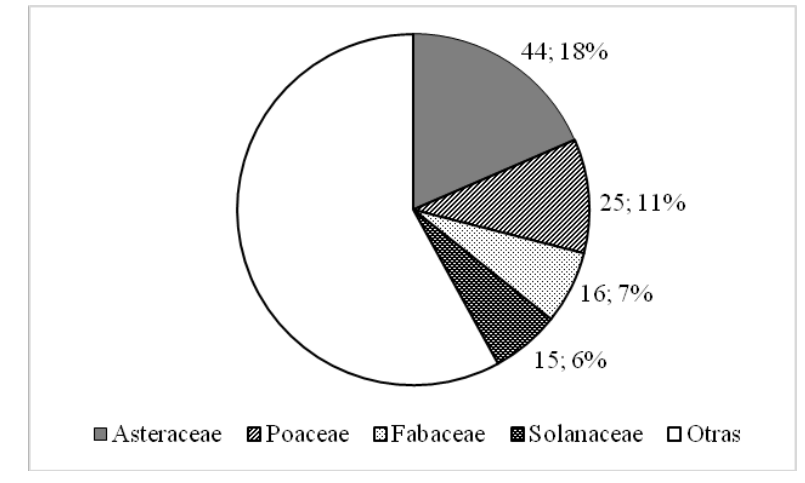

Figura 2. Riqueza de especies por familia.

especies de mayor importancia cultural. Alnus acuminata cuenta con 45 registros como combustible, Iochroma umbellatum es utilizada como medicinal (30 reportes de uso), Polylepis racemosa como combustible (40 reportes de uso), Minthostachys mollises reportada casi exclusiva como medicinal (70 reportes de uso) y Juglans neotropica es empleada como material (33 reportes de uso).

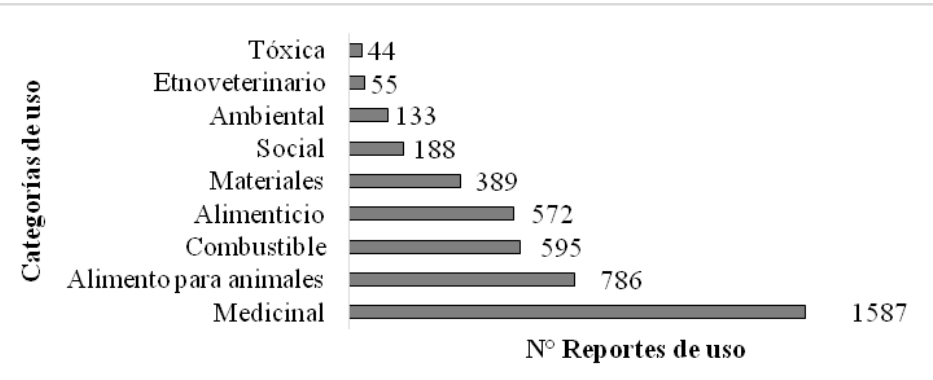

Figura 3. Número de reportes de uso por categorías.

En la Tabla 3 se describen los usos por categorías de las 10 especies más importantes para los pobladores del distrito de Pamparomás.

Tabla 2. Especies silvestres de mayor Importancia Cultural. MED = Medicinal, APA = Alimento para animales, $\mathrm{COM}=$ Combustible, $\mathrm{ALI}=$ Alimenticio, $\mathrm{MAT}=$ Materiales, $\mathrm{SOC}=$ Social, $\mathrm{AMB}=$ Ambiental, ETN = Etnoveterinario, RU = Reportes de uso, IC = Importancia Cultural.

\begin{tabular}{|c|c|c|c|c|c|c|c|c|c|c|c|}
\hline Orden & Especie & MED & APA & COM & ALI & MAT & SOC & AMB & ETN & $\mathbf{R U}$ & IC \\
\hline 1 & Alnus acuminata & 20 & 5 & 45 & & 40 & & 7 & & 117 & 0,58 \\
\hline 2 & Iochroma umbellatum & 30 & 1 & 29 & 1 & 12 & 20 & 1 & & 94 & 0,47 \\
\hline 3 & Polylepis racemosa & 15 & 5 & 40 & & 17 & & 15 & & 92 & 0,46 \\
\hline 4 & Minthostachys mollis & 70 & 11 & 1 & 7 & & & & & 89 & 0,44 \\
\hline 5 & Juglans neotropica & 15 & & 18 & 18 & 33 & & & & 84 & 0,42 \\
\hline 6 & Rorippa nasturtium-aquaticum & 33 & 7 & & 42 & & & & & 82 & 0,41 \\
\hline 7 & Ophryosporus peruvianus & 33 & 5 & 22 & & 8 & & & 8 & 76 & 0,38 \\
\hline 8 & Brassica rapa & 14 & 17 & & 42 & & & & & 73 & 0,36 \\
\hline 9 & Schinus molle & 42 & & 8 & & 22 & & & & 72 & 0,36 \\
\hline 10 & Baccharis latifolia & 16 & 12 & 27 & 2 & 14 & & & & 71 & 0,35 \\
\hline
\end{tabular}




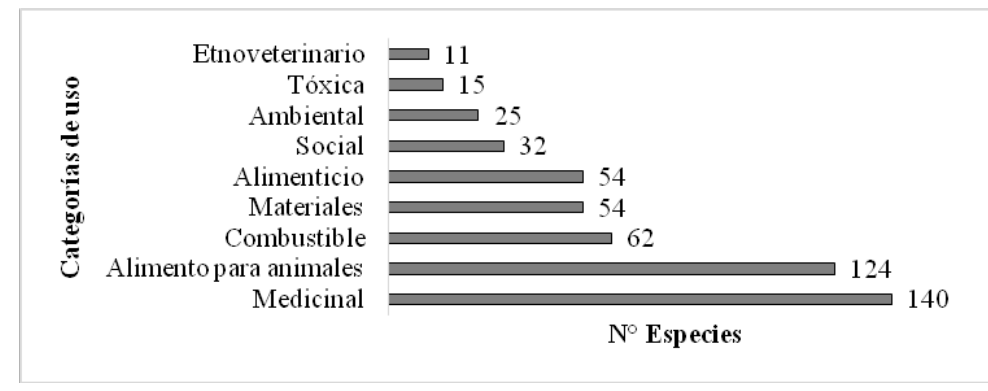

Figura 4. Riqueza de especies por categoría de uso.

\section{Discusión.}

La familia botánica con mayor riqueza de especies es Asteraceae. Ello coincide con estudios previos realizados en Pamparomás por Albán (1998) y Castañeda (2011, 2014).

El 99\% de las especies silvestres útiles cuentan por lo menos con un nombre vernáculo en idioma Quechua (Apéndice 2). Esto se debe a que por lo general los pobladores asignan nombres a las plantas que usan, por lo que es más probable que una planta que tiene nombre vernáculo tenga un uso en relación a otra que no tiene nombre local; incluso en algunos casos se puede apreciar que el poblador andino nombra a las plantas en base a los usos que le asigna, por ejemplo en la especie Ageratina sternbergiana el nombre vernáculo "warmiyaa" significa para la mujer y es empleada para evitar los achaques postparto de las mujeres, también en Vicia andicola el nombre vernáculo "haka mirasiq" significa reprodutor de cuyes y es empleada como forraje de cuyes bajo la creencia de que al comerlas estos se reproducen más.

La categoría de uso Medicinal presentó los mayores reportes de uso, seguida por Alimento para animales y Combustible (Figura 3). De manera

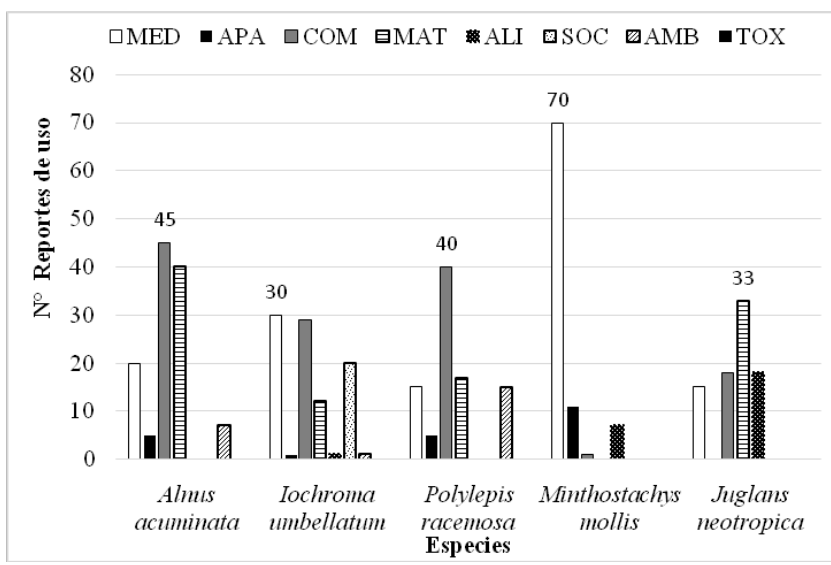

Figura 5. Categorías de uso de las especies silvestres de mayor Importancia Cultural.MED = Medicinal, APA = Alimento para animales, $\mathrm{COM}=$ Combustible, $\mathrm{MAT}=$ Materiales, $\mathrm{ALI}=$ Alimenticio, $\mathrm{SOC}=$ Social, $\mathrm{AMB}=$ Ambiental, TOX = Tóxica. similar, la categoría Medicinal tuvo la mayor riqueza de especies, seguida de Alimento para animales y Combustible (Figura 4). Trabajos previos coinciden en posicionar a la categoría Medicinal en primer lugar (Albán, 1998; Castañeda, 2011 y 2014); sin embargo, llama la atención que la categoría Alimento para animales ocupe el segundo lugar, pues en el trabajo de Albán (1998) se ubicaba en cuarto lugar. Esto se debe al mayor esfuerzo de entrevistas realizadas en los caserío de Cajabamba alta (Castañeda, 2011) y el centro poblado de Pisha (Castañeda, 2014), donde se asientan sociedades pastoriles tradicionales cuya actividad económica principal es la crianza de ganado ovino, caprino y animales menores la cual se lleva a cabo mediante prácticas de pastoreo y forrajeo de las plantas silvestres.

Albán (1998) reportó a la categoría Alimenticio en segundo lugar en cuanto a riqueza de especies, mientras que Castañeda (2014) la reporta en la tercera posición. Sin embargo, en el estudio realizado esta categoría de subsistencia se encuentra en la cuarta posición, lo cual podría evidenciar que las personas están perdiendo dependencia de las plantas silvestres que usaban de forma tradicional como alimento debido al acceso a alimentos foráneos.

Las especies más relevantes para la población Pamparomina son: Alnus acuminata (Betulaceae), Iochroma umbellatum (Solanaceae) y Polylepis racemosa (Rosaceae). Estas especies deberían tener prioridad en cuanto a conservación y manejo sostenible, más aun teniendo en cuenta que Alnus acuminata es considerada Vulnerable tanto por el Decreto Supremo 043-2006-AG (MINAG, 2006). Asimismo, Polylepis racemosa es reportada En Peligro Crítico por el Decreto Supremo 043-2006AG y como Vulnerable por la lista roja de la IUCN (World Conservation Monitoring Centre, 1998). Además, cabe mencionar que son especies leñosas y bastante visibles. Esto cobra importancia porque se está comprobando lo planteado en investigaciones previas (Pardo-deSantayana et al., 2006; Tardío \& Pardo-deSantayana, 2008) donde se indica que las plantas más complejas, tales como árboles y arbustos, tienen mayor probabilidad de ser útiles que las plantas herbáceas.

Alnus acuminata es la especie que tiene mayor número de citaciones lo cual ha determinado que tenga la Importancia Cultural más alta entre las 238 especies analizadas en este estudio. Asimismo, el hecho que Iochroma umbellatum sea la segunda especie con mayor Importancia Cultural se debe a su versatilidad, pues el número de citaciones es muy similar a la de Polylepis 
racemosa, que ocupa el tercer lugar porque tiene menor versatilidad. Así también, Minthostachys mollis y Juglans neotropica ocupan el cuarto y quinto lugar respectivamente debido a su popularidad dado que sus versatilidades son bajas (Tabla 2 y Apéndice 2).

Tabla 3. Descripción de usos de las especies silvestres de mayor Importancia Cultural.

\section{$\mathbf{N}^{0}$ Especie Descripción de los usos}

Medicinal: Se frotan las hojas con "untu" (grasa de animales como la gallina) y se pegan en las zonas inflamadas o acaloradas del cuerpo contra el dolor muscular, también se amarra la frente con las hojas frescas contra el dolor de cabeza por calor. Alimento para animales: Las ramas tiernas son consumidas por animales domésticos como las cabras y burros. Combustible: La madera y las ramas secas sirven de leña. Materiales: A Alnus partir de su madera se elaboran acuminata diferentes herramientas como la "taklla" (arado), mango del "ishwi" (pequeña picota de metal) y mazo; además la planta entera es utilizada como escoba en la "parva" (cosecha). La madera sirve como umbral y columna en la construcción de casas. Se tiñen con las hojas frescas obteniéndose un color marrón o "abano" (color camello). Ambiental: La planta completa sirve de cerco vivo cuando se siembra al borde de chacras, huertos familiares o caminos.

Medicinal: Se amarra la frente contra el dolor de cabeza por susto. Se toma la infusión de sus ramas, junto con las hojas de Baccharis genistelloides para abortar. Alimento para animales: Las hojas tiernas son consumidas por animales como la cabra, la vaca y el burro. Combustible: Los tallos secos sirven de leña. Alimenticio: Las hojas se echan al pelado de trigo para ayudar en la cocción. Materiales: Los tallos sirven para elaborar "pirwa" (madera 2 Iochroma delgada que sirve para hilar) y "kaalla" umbellatum (huso o madero delgado que sirve para amarrar la lana del hilado). Las hojas se echan al pelado de trigo para ayudar en la cocción. Social: Se calientan los frutos al fuego y se "shuukma" (práctica ritual en la cual se pasa el cuerpo de una persona con plantas aromáticas, invocándose a los santos mediante rezos y cantos). Ambiental: La planta completa sirve de cerco vivo cuando se siembra al borde de chacras o caminos.
Tabla 3. Descripción de usos de las especies silvestres de mayor Importancia Cultural.

\section{$N^{o}$ Especie Descripción de los usos}

Medicinal: Con la infusión de las ramas se realizan baños y lavados contra el sobreparto; además se toma la infusión de las ramas tiernas contra problemas respiratorios. Alimento para animales: Las hojas tiernas son consumidas por algunos animales como Polylepis las cabras y los burros. Combustible: racemosa Las ramas secas sirven de leña. Materiales: Los tallos sirven para elaborar "taklla" y mazo. A partir del tronco se elaboran maderas que sirven para la construcción de casas y puentes. Ambiental: La planta completa sirve de cerco vivo cuando se siembra al borde de chacras o caminos.

Medicinal: Se toma la infusión de las ramas tiernas contra resfríos, dolor de barriga por gases (anti flatulento) y para evitar los antojos de las mujeres embarazadas. Alimento para

4 Minthostachys animales: Las hojas tiernas son mollis consumidas por algunos animales como cuyes y ovejas. Combustible: Las ramas secas se utilizan como leña para encender el fuego. Alimenticio: Sus hojas aromáticas se emplean como condimento en la preparación se sopas. Medicinal: Con el hervor de las ramas se hacen baños para aliviar dolores musculares causados por el frío. Combustible: Las ramas secas sirven de leña. Alimenticio: La semilla es comestible. Materiales: Se tiñen con las hojas frescas obteniéndose un color marrón o "abano". La madera se emplea en la construcción de casas y para elaborar instrumentos musicales como la guitarra.

Medicinal: El jugo o sumo de las hojas chancadas se toma contra gastritis, úlceras, inflamación, dolor de cabeza, infección, escorbuto ("shama"), enfermedades del riñón e hígado.

Alimenticio: Se echa limón y sal a las 6 nasturtium - hojas tiernas y frescas para preparar aquaticum una ensalada que se come con papa sancochada; también es posible sancochar las hojas y hacer un guiso ("pichu") que se come con papa o cancha. Alimento para animales: Las ramas tiernas son consumidas por animales como el cerdo. 
Tabla 3. Descripción de usos de las especies silvestres de mayor Importancia Cultural.

\begin{tabular}{ll}
$\mathbf{N}^{\circ}$ Especie \\
\hline 7 & $\begin{array}{l}\text { Ophryosporus } \\
\text { peruvianus }\end{array}$
\end{tabular}

Descripción de los usos

Medicinal: Con las hojas frescas calentadas en fuego se amarra la zona afectada o se hacen frotaciones contra golpes, dislocaduras o fracturas de personas o animales. Alimento para animales: Las ramas tiernas sirven de comida a los animales como el burro, la oveja y la vaca. Combustible: Las ramas secas sirven de combustible. Materiales: Con la madera se elabora "pirwa” y "kaalla” para hilar; además la planta entera es utilizada como escoba en la cosecha ("parva”).

Medicinal: Se chanca la planta entera y se pone sobre la frente ("chakta”) para aliviar el dolor de cabeza (“tabardillo"). Alimenticio: Las hojas y flores se sancochan y aderezan para

8 Brassica rapa para la preparación de un plato típico conocido como "yuyo" o "pichu” que se come con cancha, papa y ají. Alimento para animales: La planta fresca sirve de alimento a animales domésticos como cuyes, conejos, ovejas y vacas.

Medicinal: Se hierven las hojas para realizar baños contra el "mal de aire". Combustible: Las ramas secas sirven de leña. Materiales: A partir de su madera se elaboran algunas

9 Schinus molle herramientas como "ishwi". Las hojas sirven para realizar teñidos obteniéndose una coloración verde amarillenta. La madera se emplea en construcción de casas. El látex es utilizado como goma.

Medicinal: Con las hojas tiernas se amarra la cabeza contra el frío, igualmente se soba la cabeza con las hojas calientes contra "mal de aire", también se hacen frotaciones con las hojas tostadas en caso de golpes o dislocaduras. Alimento para animales: Las ramas tiernas sirven de comida a animales mayores como vacas y burros. Combustible: Las ramas secas sirven de leña. Alimenticio: Con las hojas se tapa el maíz antes que germine para mejorar el sabor a la chicha de jora. Materiales: Las hojas sirven para realizar teñidos obteniéndose una coloración verdosa, también se pegan sobre heridas pequeñas para protegerlas.

El uso principal de Alnus acuminata es como Combustible, mientras que Iochroma umbellatum es utilizada como Medicinal, Polylepis racemosa como Combustible y Minthostachys mollis es reportada casi exclusivamente como Medicinal (Figura 5). Ello demuestra la importancia de las categorías Medicinal y Combustible en Pamparomás. Sin embargo, cabe recalcar que si bien las plantas empleadas como Alimento Para Animales no ocupan los primeros lugares en cuanto a Importancia Cultural, estas cobran importancia debido a la gran riqueza de especies empleadas para este fin (Figura 4), tal como lo demuestra el estudio realizado por Castañeda et al. (2014) para el caserío de Pisha donde se reportan 117 especies silvestres de importancia ganadera.

Cabe mencionar que Alnus acuminata, Iochroma umbellatum y Polylepis racemosa son especies leñosas y bastante visibles, esto cobra importancia porque se está comprobando lo planteado en investigaciones previas (Pardo-de-Santayana et al., 2006; Tardío y Pardo-de-Santayana, 2008) donde se indican que las plantas más complejas, tales como árboles y arbustos, tienen mayor probabilidad de ser útiles que las plantas herbáceas.

El índice de Importancia Cultural, empleado en el presente estudio, solo considera categorías de uso amplias con la finalidad de conocer las plantas más importantes para una determinada comunidad; mas no para conocer por ejemplo qué plantas medicinales son más efectivas, puesto que los usos de las especies medicinales para diferentes dolencias son contabilizados como únicos, así por ejemplo se tiene que Minthostachys mollis es reportado contra resfríos, como anti flatulento y para evitar los antojos de las mujeres embarazadas; sin embargo, las tres prescripciones están agrupadas en una única categoría Medicinal (Tabla 3). Ello puede subestimar la real significancia cultural de las especies medicinales, siendo necesario un estudio más profundo -a nivel de sub categorías de uso- que sea sensible a dichas peculiaridades.

Para conservar la flora se debe tomar en cuenta su uso e importancia dentro de la cultura del grupo humano que se estudia; pues es más adecuado conservar y manejar especies utilizadas tradicionalmente que aquellas que no lo son, debido que las primeras son más sensibles al impacto antrópico (Castañeda, 2014). En tal sentido las plantas silvestres de mayor Importancia Cultural son buenas candidatas para realizar investigaciones más minuciosas sobre la naturaleza de sus características particulares que las hacen útiles, por lo cual se sugiere que éstas sean manejadas sosteniblemente con fines de mejorar la calidad de vida de los pobladores pamparominos.

\section{Conclusiones.}

Se reportan 237 especies de plantas silvestres útiles para el distrito de Pamparomás.

Las categorías con mayor número de especies y mayores reportes de usos son Medicinal, Alimento Para Animales y Combustible. 
Las plantas silvestres de mayor Importancia Cultural son Alnus acuminata, Iochroma umbellatum y Polylepis racemosa.

La información obtenida sirve como base para estudios posteriores relacionados con temas de Botánica económica, Farmacología, Nutrición, Ecología, Manejo y Conservación de recursos vegetales.

\section{Agradecimientos.}

Al fondo de desarrollo universitario y promoción de trabajo de tesis de pregrado del VRI-UNMSM y a la ONG Aide Au Développemet Gembloux (ADG)Pamparomás por el apoyo económico brindado.

A Dora Rivera y Victoriano Rivera, quienes nos acogieron en sus viviendas y brindaron todo su apoyo durante nuestras estancias. A los directores y curadores de los Herbarios USM, CUZ, F y MO por el apoyo en la identificación de las especies. A Martha Montiel por su apoyo durante la visita al Herbario MO.

\section{Literatura citada.}

Albán J.A. 1985. Un registro de datos etnobotánicos. Boletín de Lima. 7(39): 93-96.

1998. Etnobotánica y conservación en la comunidad andina de Pamparomás Huaylas, Ancash, Perú. Tesis para optar al grado académico de Magíster en Botánica Tropical con mención en Etnobotánica, Escuela de Posgrado, Facultad de Ciencias Biológicas, Universidad Nacional Mayor de San Marcos. LimaPerú. 267 pp.

2013. Etnobotánica de rubiáceas peruanas. Tesis para optar al grado académico de Doctor en Ciencias Biológicas. Escuela de Posgrado, Facultad de Ciencias Biológicas, Universidad Nacional Mayor de San Marcos, Lima - Perú. 721 pp.

Alexiades M. 1996. Collecting Ethnobotanical Data: An Introduction to Basic Concepts and Techniques. Selected Guidelines for Ethnobotanical Research: A Fiel Manual 53-94.

Alvarado B. 2007. Plantas Medicinales de la Cordillera Negra. Revista Académica Perú Salud. 14(2): 53-63.

APG IV. 2016. An update of the Angiosperm Phylogeny Group classification for the orders and families of flowering plants: APG IV. Botanical Journal of the Linnean Society. 181: 1-20.

Castañeda R.Y. 2011. Valor de uso de las plantas silvestres en Pamparomás, Ancash. Tesis para optar al Título Profesional de Bióloga con mención en Botánica. Escuela Académico Profesional de Ciencias Biológicas, Facultad de Ciencias Biológicas, Universidad Nacional Mayor de San Marcos. Lima- Perú. 124 pp.

2014. Comparación de tres índices de significancia cultural de la flora silvestre del caserío de Pisha (Pamparomás, Ancash). Tesis para optar al grado académico de Magíster en Botánica Tropical con mención en Etnobotánica, Escuela de Posgrado, Facultad de Ciencias Biológicas, Universidad Nacional Mayor de San Marcos. Lima- Perú. 100 pp.

., Albán J.A., Gutiérrez H., Cochachin E. \&

La Torre M.I. 2014. Plantas silvestres empleadas como alimento para animales en Pisha, Ancash. Ecología Aplicada 13(2): 153-168.

Cerrate E. 1964. Manera de preparar plantas para un herbario. UNMSM. Museo de Historia Natural, Serie de Divulgación 1.

Chemisquy M.A., Giussani L.M., Scataglini M.A., Kellogg E.A. \& Morrone O. 2010. Phylogenetic studies favour the unification of Pennisetum, Cenchrus and Odontelytrum (Poaceae): A combined nuclear, plastid and morphological analysis, and nomenclatural combinations in Cenchrus. Annals of Botany. 106: 107130.

Gruca M.R., Cámara-Leret R., Macía M.J. \& Balslev H. 2014. New categories for traditional medicine in the Econ.Bot. Data Collection Standard.J Ethnopharmacol. 155: 1388-1392.

Julca F. 2009. Quechua ancashino: una mirada actual. Care Perú. Primera edición. Lima - Perú. Fondo editorial del Pedagógico San Marcos. ISBN 978-612-45425-4-1. 430 pp.

Kufer J., Förther H., Pöll E. \& Heinrich M. 2005.Historical and Modern Medicinal Plant Uses-The Example of the Chorti Maya and Ladinos in Eastern Guatemala. J Ethnopharmacol. 57(9):1127-1152.

Levy T.S. \& Aguirre J. 1999. Conceptuación etnobotánica (experiencia de un estudio en la Lacandona). Revista de Geografía Agrícola 29: 83-114.

Lodé J. \& Pino G. 2008. Agave cordillerensis J. Lodé \& G. Pino Una nueva especie de América del Sur. Int. Cact. Advent. 77: 13.

Martínez M. 1991. La etnobotánica en Latinoamérica. Memorias III Simposio Colombiano de Etnobotánica, Cali, INCIVA.1-14 pp.

Melchor R.B. 2016. Etnobotánica y diversidad de la familia Leguminosae Juss. en la cuenca del río Nepeña (Ancash, Perú). Tesis para optar al Título Profesional de Bióloga con mención en Botánica. Escuela Académico Profesional de Ciencias Biológicas, Facultad de Ciencias Biológicas, Universidad Nacional Mayor de San Marcos. Lima- Perú. 122 pp.

MINAG (Ministerio de Agricultura). 2006. Categorización de especies amenazadas de flora silvestre D.S.N ${ }^{\circ}$ 043AG-2006.

Moreno Gu. 1966. Estudio Monográfico del Distrito de Pamparomas. Tesis de grado. Instituto nacional de perfeccionamiento magisterial. Huaraz, Ancash. ISBN. $84-922495-2-8$.

Moreno Ga. 1989. Proyecto de Desarrollo Integral de Pamparomás. Concytec, Lima.

Paniagua N., Macía M.J. \& Cámara R. 2010. Toma de datos etnobotánicos de palmeras y variables socioeconómicas en comunidades rurales. Ecol. Boliv. 45(3): 44-68.

Pardo-De-Santayana M., Tardío J., Heinrich M., Touwaide A. \& Morales R. 2006. Plants in the Works of Cervantes. Economic Botany. 60(2):159-181.

Phillips O. \& Gentry A. 1993. The useful plants of Tambopata, Peru: I. Statistical hypotheses tests with a new quantitative technique. Economic Botany. 47(1): 15-32.

Reyes-García V., Huanca T., Vadez V., Leonard W., Wilkie D. 2006. Cultural, practical, and economic value of wild plants: A quantitative study in the Bolivian Amazon. Economic Botany. 60(1): 62-74 
Tardío J. \& Pardo-De-Santayana M. 2008. Cultural Importance Indices: A Comparative Analysis Based on the Useful Wild Plants of Southern Cantabria (Northern Spain). Economic Botany. 62(1): 24-39.

Tropicos. 2016. Tropicos.org, Missouri Botanical Garden. http://www.tropicos.org
World Conservation Monitoring Centre. 1998. Polylepis racemosa. The IUCN Red List of Threatened Species 1998: $\quad$ e.T32289A9687283. http://dx.doi.org/10.2305/IUCN.UK.1998.RLTS.T32289 A968728.

Apéndice 1. Ficha para recopilar información etnobotánica.

\section{ENCUESTA SEMIESTRUCTURADA}

\section{DATOS DEL INFORMANTE:}

Nombre:

Edad: .

Lugar de nacimiento:

DATOS BOTÁNICOS:

Nombre científico:

Familia:

DATOS ADICIONALES:

Fecha de la encuesta:

Código de la muestra:

1. ¿CUÁL ES EL NOMBRE LOCAL DE LA PLANTA?

2. ¿PARA QUÉ UTILIZA LA PLANTA?
a) Medicinal
b) Alimento para animales
c) Combustible
d) Alimenticio
e) Materiales
f) Social
g) Ambiental
h) Etnoveterinario
i) Tóxica

j) Otros

b) Tallo

a) Raíz

d) Flores

e) Frutos

g) Corteza

h) Planta entera

c) Hojas

f) Semillas

4. ¿CÓMO LA UTILIZA?

i) Otras. 
Apéndice 2. Lista de la flora silvestre útil del distrito de Pamparomás, Ancash. MED = Medicinal, APA = Alimento para animales, $\mathrm{COM}=$ Combustible, $\mathrm{ALI}=$ Alimenticio, $\mathrm{MAT}=$ Materiales, $\mathrm{SOC}=$ Social, $\mathrm{AMB}=\mathrm{Ambiental}, \mathrm{TOX}=$ Tóxica, ETN = Etnoveterinario, RU = Reportes de uso, IC = Importancia Cultural.

\begin{tabular}{|c|c|c|c|c|c|c|c|c|c|c|c|c|c|}
\hline Familia & Especie & $\begin{array}{l}\text { Nombre } \\
\text { vernáculo }\end{array}$ & $\begin{array}{l}\text { M } \\
\text { E } \\
D\end{array}$ & $\begin{array}{l}\text { AP } \\
\text { A }\end{array}$ & $\begin{array}{l}\mathrm{CO} \\
\mathrm{M}\end{array}$ & $\begin{array}{l}\text { A } \\
\text { LI }\end{array}$ & $\begin{array}{l}\text { M } \\
\text { AT }\end{array}$ & $\begin{array}{l}\mathrm{SO} \\
\mathrm{C}\end{array}$ & $\begin{array}{l}\mathrm{AM} \\
\mathrm{B}\end{array}$ & $\begin{array}{l}\text { TO } \\
\mathrm{X}\end{array}$ & $\begin{array}{l}\mathrm{ET} \\
\mathrm{N}\end{array}$ & RU & IC \\
\hline $\begin{array}{l}\text { Acanthacea } \\
\text { e }\end{array}$ & Dicliptera multiflora (Ruiz \& Pav.) Juss. & $\begin{array}{l}\text { Kinwaksh, } \\
\text { kinwua }\end{array}$ & 10 & 5 & & 17 & & 7 & & & & 39 & $\begin{array}{r}0,1 \\
9\end{array}$ \\
\hline Adoxaceae & $\begin{array}{l}\text { Sambucus mexicana var. bipinnata } \\
\text { (Schltdl. \& Cham.) Schwer. }\end{array}$ & $\begin{array}{l}\text { Sauquillo, } \\
\text { sauco }\end{array}$ & 3 & & & & & & & & & 3 & $\begin{array}{r}0,0 \\
1\end{array}$ \\
\hline Adoxaceae & Sambucus peruviana Kunth & $\begin{array}{l}\text { Rayán, arrayán, } \\
\text { sauco }\end{array}$ & 15 & & 10 & 10 & 13 & & 1 & & & 49 & $\begin{array}{r}0,2 \\
4\end{array}$ \\
\hline Adoxaceae & $\begin{array}{l}\text { Viburnum mathewsii (Oerst.) Killip \& } \\
\text { A.C. Sm. }\end{array}$ & Putska & & & 1 & & & & & & & 1 & $\begin{array}{r}0,0 \\
0\end{array}$ \\
\hline $\begin{array}{l}\text { Alstroemeri } \\
\text { aceae }\end{array}$ & Alstroemeria chorillensis Herb. & Korpus wayta & & & & & & & 4 & & & 4 & $\begin{array}{r}0,0 \\
2\end{array}$ \\
\hline $\begin{array}{l}\text { Amarantha } \\
\text { ceae }\end{array}$ & Alternanthera porrigens (Jacq.) Kuntze & $\begin{array}{l}\text { Cruz qiru, palo } \\
\text { de cruz }\end{array}$ & 17 & 4 & & & & 16 & & & & 37 & $\begin{array}{r}0,1 \\
8\end{array}$ \\
\hline $\begin{array}{l}\text { Amarantha } \\
\text { ceae }\end{array}$ & Amaranthus hybridus L. & $\begin{array}{l}\text { Yuyo, wallpa } \\
\text { yuyo, aataqo, } \\
\text { hitqa }\end{array}$ & 8 & 4 & & 22 & & & & & & 34 & $\begin{array}{r}0,1 \\
7\end{array}$ \\
\hline $\begin{array}{l}\text { Amarantha } \\
\text { ceae }\end{array}$ & Chenopodium murale L. & Lichqanya & 3 & 1 & & & & & & & & 4 & $\begin{array}{r}0,0 \\
2\end{array}$ \\
\hline $\begin{array}{l}\text { Amarantha } \\
\text { ceae }\end{array}$ & $\begin{array}{l}\text { Dysphania ambrosioides (L.) Mosyakin \& } \\
\text { Clemants }\end{array}$ & $\begin{array}{l}\text { Kaamash, } \\
\text { paico, } \\
\text { chichanga }\end{array}$ & 34 & & & 5 & & & & & & 39 & $\begin{array}{r}0,1 \\
9\end{array}$ \\
\hline $\begin{array}{l}\text { Amarantha } \\
\text { ceae }\end{array}$ & $\begin{array}{l}\text { Guilleminea densa (Willd. ex Schult.) } \\
\text { Moq. }\end{array}$ & Kushpi & 9 & 9 & & & & & & & & 18 & $\begin{array}{r}0,0 \\
9\end{array}$ \\
\hline $\begin{array}{l}\text { Amaryllida } \\
\text { ceae }\end{array}$ & Ismene amancaes (Ruiz \& Pav.) Herb. & $\begin{array}{l}\text { Amancay, } \\
\text { weshu }\end{array}$ & & & & & & & 6 & & & 6 & $\begin{array}{r}0,0 \\
3\end{array}$ \\
\hline $\begin{array}{l}\text { Anacardiac } \\
\text { eae }\end{array}$ & Schinus molle L. & Molle & 42 & & 8 & & 22 & & & & & 72 & $\begin{array}{r}0,3 \\
6\end{array}$ \\
\hline Apiaceae & Arracacia xanthorrhiza Bancr. & $\begin{array}{l}\text { Araqacha, } \\
\text { raqacha, tuna } \\
\text { raqacha }\end{array}$ & 1 & & & 1 & & & & & & 2 & $\begin{array}{r}0,0 \\
1\end{array}$ \\
\hline Apiaceae & Azorella crenata (Ruiz \& Pav.) Pers. & Patsapa maquin & & & & 1 & & 4 & & & & 5 & $\begin{array}{r}0,0 \\
2\end{array}$ \\
\hline $\begin{array}{l}\text { Apocynace } \\
\text { ae }\end{array}$ & Cynanchum formosum N.E.Br. & $\begin{array}{l}\text { Tijerillas, } \\
\text { tijeretas }\end{array}$ & 10 & & & 5 & & & & & & 15 & $\begin{array}{r}0,0 \\
7\end{array}$ \\
\hline Araceae & Zantedeschia aethiopica (L.) Spreng. & Cartucho & & & & & & & 22 & & & 22 & $\begin{array}{r}0,1 \\
1\end{array}$ \\
\hline Araliaceae & Oreopanax oroyanus Harms & Mántak, pati & & & 6 & & 2 & & & & & 8 & $\begin{array}{r}0,0 \\
4\end{array}$ \\
\hline $\begin{array}{l}\text { Asparagace } \\
\text { ae }\end{array}$ & Agave cordillerensis Lodé \& Pino & Penka, pinka & & & 3 & & 25 & & 10 & & 3 & 41 & $\begin{array}{r}0,2 \\
0\end{array}$ \\
\hline $\begin{array}{l}\text { Asparagace } \\
\text { ae }\end{array}$ & Furcraea andina Trel. & $\begin{array}{l}\text { Penka, } \\
\text { maguey, toco }\end{array}$ & & & & & 4 & & & & & 4 & $\begin{array}{r}0,0 \\
2\end{array}$ \\
\hline $\begin{array}{l}\text { Asparagace } \\
\text { ae }\end{array}$ & Trihesperus glaucus (Ruiz \& Pav.) Herb. & Sakuapa aran & & & & 1 & & & & & & 1 & $\begin{array}{r}0,0 \\
0\end{array}$ \\
\hline Asteraceae & Achyrocline alata (Kunth) DC. & Lichupa rinrin & 6 & & & & & & & & & 6 & $\begin{array}{r}0,0 \\
3\end{array}$ \\
\hline Asteraceae & $\begin{array}{l}\text { Ageratina sternbergiana (DC.) R.M.King } \\
\text { \& H.Rob. }\end{array}$ & Warmiyaa & 34 & 3 & & & & & & & & 37 & $\begin{array}{r}0,1 \\
8\end{array}$ \\
\hline Asteraceae & Ambrosia arborescens Mill. & Marco, marcuu & 43 & 5 & 5 & & 1 & & & & & 54 & $\begin{array}{r}0,2 \\
7\end{array}$ \\
\hline Asteraceae & Antennaria linearifolia Wedd. & Coca silvestre & & & & & & 1 & & & & 1 & $\begin{array}{r}0,0 \\
0\end{array}$ \\
\hline Asteraceae & $\begin{array}{l}\text { Aristeguietia discolor (DC.) R.M.King \& } \\
\text { H.Rob. }\end{array}$ & $\begin{array}{l}\text { Tsayanco, } \\
\text { killua }\end{array}$ & 1 & 1 & 12 & & & & & & & 14 & $\begin{array}{r}0,0 \\
7\end{array}$ \\
\hline Asteraceae & Artemisia absinthium L. & Ajenko & 1 & & & & & & & & & 1 & $\begin{array}{r}0,0 \\
0\end{array}$ \\
\hline Asteraceae & Baccharis boliviensis (Wedd.) Cabrera & $\begin{array}{l}\text { Ucushpa } \\
\text { chupan }\end{array}$ & & & 1 & & 2 & & & & & 3 & $\begin{array}{r}0,0 \\
1\end{array}$ \\
\hline Asteraceae & Baccharis genistelloides (Lam.) Pers. & $\begin{array}{l}\text { Chinchimallii, } \\
\text { karkeha, }\end{array}$ & 29 & 1 & & & & 3 & & & & 33 & $\begin{array}{r}0,1 \\
6\end{array}$ \\
\hline
\end{tabular}


Apéndice 2. Lista de la flora silvestre útil del distrito de Pamparomás, Ancash. MED = Medicinal, APA = Alimento para animales, $\mathrm{COM}=$ Combustible, $\mathrm{ALI}=$ Alimenticio, $\mathrm{MAT}=$ Materiales, $\mathrm{SOC}=$ Social, $\mathrm{AMB}=\mathrm{Ambiental}, \mathrm{TOX}=$ Tóxica, ETN = Etnoveterinario, RU = Reportes de uso, IC = Importancia Cultural.

\begin{tabular}{|c|c|c|c|c|c|c|c|c|c|c|c|c|c|}
\hline Familia & Especie & $\begin{array}{l}\text { Nombre } \\
\text { vernáculo }\end{array}$ & $\begin{array}{l}\text { M } \\
\text { E } \\
\text { D }\end{array}$ & $\begin{array}{l}\text { AP } \\
\text { A }\end{array}$ & $\begin{array}{l}\mathrm{CO} \\
\mathrm{M}\end{array}$ & $\begin{array}{l}\text { A } \\
\text { LI }\end{array}$ & $\begin{array}{l}\text { M } \\
\text { AT }\end{array}$ & $\begin{array}{l}\mathrm{SO} \\
\mathrm{C}\end{array}$ & $\begin{array}{l}\mathrm{AM} \\
\mathrm{B}\end{array}$ & $\begin{array}{l}\text { TO } \\
\mathrm{X}\end{array}$ & $\begin{array}{l}\text { ET } \\
N\end{array}$ & RU & IC \\
\hline & & esquinado & & & & & & & & & & & \\
\hline Asteraceae & Baccharis latifolia (Ruiz \& Pav.) Pers. & Tsillka, chillka & 16 & 12 & 27 & 2 & 14 & & & & & 71 & $\begin{array}{r}0,3 \\
5\end{array}$ \\
\hline Asteraceae & Baccharis tomentosa Thunb. & $\begin{array}{l}\text { Paaqllash } \\
\text { monte }\end{array}$ & & & 3 & & & & & & & 3 & $\begin{array}{r}0,0 \\
1\end{array}$ \\
\hline Asteraceae & Baccharis tricuneata (L.f.) Pers. & Llinllish, taya & 6 & 1 & 51 & & 9 & & & & & 67 & $\begin{array}{r}0,3 \\
3\end{array}$ \\
\hline Asteraceae & Bidens pilosa L. & $\begin{array}{l}\text { Shillku, yuraq } \\
\text { shillku, chilco, } \\
\text { amor seco }\end{array}$ & 22 & 24 & & 3 & & 1 & & & & 50 & $\begin{array}{r}0,2 \\
5\end{array}$ \\
\hline Asteraceae & Chionopappus benthamii S.F.Blake & Kuruputska & & 8 & & 9 & & & & & & 17 & $\begin{array}{r}0,0 \\
8\end{array}$ \\
\hline Asteraceae & Chuquiraga spinosa Less. & Huamanpinta & 32 & & & & & & & & & 32 & $\begin{array}{r}0,1 \\
6\end{array}$ \\
\hline Asteraceae & $\begin{array}{l}\text { Cronquistianthus chamaedrifolius (Kunth) } \\
\text { R.M.King \& H.Rob. }\end{array}$ & Yana munti & & & 6 & & 3 & & & & & 9 & $\begin{array}{r}0,0 \\
4\end{array}$ \\
\hline Asteraceae & Dasyphyllum ferox (Wedd.) Cabrera & Quntsi & 1 & & 7 & & 1 & & 2 & & & 11 & $\begin{array}{r}0,0 \\
5\end{array}$ \\
\hline Asteraceae & $\begin{array}{l}\text { Dendrophorbium usgorense (Cuatrec.) } \\
\text { C.Jeffrey }\end{array}$ & Ruquruwaa & 3 & & & & 1 & & & & & 4 & $\begin{array}{r}0,0 \\
2\end{array}$ \\
\hline Asteraceae & Gnaphalium americanum Mill. & $\begin{array}{l}\text { Allqupakallun, } \\
\text { lengua de perro }\end{array}$ & 21 & 3 & & & & & & & & 24 & $\begin{array}{r}0,1 \\
2\end{array}$ \\
\hline Asteraceae & Gynoxys caracensis Muschl. & Limbar & 1 & & & & & & & & & 1 & $\begin{array}{r}0,0 \\
0\end{array}$ \\
\hline Asteraceae & Heliopsis buphthalmoides (Jacq.) Dunal & $\begin{array}{l}\text { Qaqush, } \\
\text { wishka wayta, } \\
\text { qoha qora }\end{array}$ & 1 & & & & & & & & & 1 & $\begin{array}{r}0,0 \\
0\end{array}$ \\
\hline Asteraceae & $\begin{array}{l}\text { Hypochaeris taraxacoides (Meyen \& } \\
\text { Walp.) Ball }\end{array}$ & $\begin{array}{l}\text { Chicoria de la } \\
\text { puna }\end{array}$ & 1 & & & & & & & & & 1 & $\begin{array}{r}0,0 \\
0\end{array}$ \\
\hline Asteraceae & Jungia schuerae Harling & $\begin{array}{l}\text { karamatas, } \\
\text { matico, } \\
\text { matsacraa }\end{array}$ & 42 & 13 & 3 & & & & & & & 58 & $\begin{array}{r}0,2 \\
9\end{array}$ \\
\hline Asteraceae & $\begin{array}{l}\text { Munnozia hastifolia (Poepp.) H.Rob. \& } \\
\text { Brettell }\end{array}$ & Yolae wayta & & 1 & & & & & & & & 1 & $\begin{array}{r}0,0 \\
0\end{array}$ \\
\hline Asteraceae & Mutisia acuminata Ruiz \& Pav. & $\begin{array}{l}\text { Matzacraa, } \\
\text { Matzecraa, } \\
\text { Santiago, San } \\
\text { Santiago }\end{array}$ & 21 & 3 & 2 & & 3 & 9 & & & & 38 & $\begin{array}{r}0,1 \\
9\end{array}$ \\
\hline Asteraceae & $\begin{array}{l}\text { Ophryosporus peruvianus R.M.King \& } \\
\text { H.Rob. }\end{array}$ & $\begin{array}{l}\text { Kipiksaa, } \\
\text { sayanco }\end{array}$ & 33 & 5 & 22 & & 8 & & & & 8 & 76 & $\begin{array}{r}0,3 \\
8\end{array}$ \\
\hline Asteraceae & Pappobolus acutifolia (S.F.Blake) Panero & Latak suncho & 1 & & & & & & & & & 1 & $\begin{array}{r}0,0 \\
0\end{array}$ \\
\hline Asteraceae & $\begin{array}{l}\text { Paracalia jungioides (Hook. \& Arn.) } \\
\text { Cuatrec. }\end{array}$ & Marirkaa & 1 & & 12 & 8 & & & & & & 21 & $\begin{array}{r}0,1 \\
0\end{array}$ \\
\hline Asteraceae & Paranephelius bullatus A.Gray ex Wedd. & Panaas & & 6 & & & & & & & & 6 & 0,0 \\
\hline Asteraceae & Perezia multiflora (Humb. \& Bonpl.) Less. & Escorsonera & 19 & & & & & & & & & 19 & 0,0 \\
\hline Asteraceae & Schkuhria pinnata (Lam.) Kuntze ex Thell. & Ayaq pitsana & 11 & & & & 3 & & & & & 14 & 0,0 \\
\hline Asteraceae & Senecio albaniae H.Beltrán & $\begin{array}{l}\text { Karuash, } \\
\text { kalluash, wayta }\end{array}$ & & 3 & & & & & 1 & & & 4 & $\begin{array}{r}0,0 \\
2\end{array}$ \\
\hline Asteraceae & Senecio campanellifer Cuatrec. & Alalaq munti & & & 4 & & 1 & & & & & 5 & $\begin{array}{r}0,0 \\
2\end{array}$ \\
\hline Asteraceae & Senecio canescens Bernh.ex DC. & Luichupa rinrin & 24 & & & & & & & & & 24 & $\begin{array}{r}0,1 \\
2\end{array}$ \\
\hline Asteraceae & Senecio sp. (R. Castañeda 623) & $\begin{array}{l}\text { Upituckru, } \\
\text { upitucllu, }\end{array}$ & 2 & 4 & 4 & & & & & & & 10 & $\begin{array}{r}0,0 \\
5\end{array}$ \\
\hline
\end{tabular}


Apéndice 2. Lista de la flora silvestre útil del distrito de Pamparomás, Ancash. MED = Medicinal, APA = Alimento para animales, $\mathrm{COM}=$ Combustible, $\mathrm{ALI}=$ Alimenticio, $\mathrm{MAT}=$ Materiales, $\mathrm{SOC}=$ Social, $\mathrm{AMB}=$ Ambiental, $\mathrm{TOX}=$ Tóxica, ETN = Etnoveterinario, RU = Reportes de uso, IC = Importancia Cultural.

\begin{tabular}{|c|c|c|c|c|c|c|c|c|c|c|c|c|c|}
\hline Familia & Especie & $\begin{array}{l}\text { Nombre } \\
\text { vernáculo }\end{array}$ & $\begin{array}{l}M \\
E \\
D\end{array}$ & $\begin{array}{l}\text { AP } \\
\text { A }\end{array}$ & $\begin{array}{l}\mathrm{CO} \\
\mathrm{M}\end{array}$ & $\begin{array}{l}\text { A } \\
\text { LI }\end{array}$ & $\begin{array}{l}\text { M } \\
\text { AT }\end{array}$ & $\begin{array}{l}\text { SO } \\
\text { C }\end{array}$ & $\begin{array}{l}\mathrm{AM} \\
\mathrm{B}\end{array}$ & $\begin{array}{l}\text { TO } \\
\mathrm{X}\end{array}$ & $\begin{array}{l}\mathrm{ET} \\
\mathrm{N}\end{array}$ & RU & IC \\
\hline & & $\begin{array}{l}\text { upiipatucllum, } \\
\text { sucumonte }\end{array}$ & & & & & & & & & & & \\
\hline Asteraceae & Smallanthus fruticosus (Benth.) H.Rob. & $\begin{array}{l}\text { Putaqa, } \\
\text { yanaputaqa, } \\
\text { putka }\end{array}$ & 21 & 1 & 19 & & 3 & & & & & 44 & $\begin{array}{r}0,2 \\
2\end{array}$ \\
\hline Asteraceae & Sonchus asper (L.) Hill & $\begin{array}{l}\text { Kgaña, } \\
\text { kashakgaña } \\
\text { macho, cerraja }\end{array}$ & 21 & 10 & & & & & & & & 31 & $\begin{array}{r}0,1 \\
5\end{array}$ \\
\hline Asteraceae & Sonchus oleraceus (L.) L. & $\begin{array}{l}\text { Kashakgaña, } \\
\text { hembra, cerraja } \\
\text { kgaña }\end{array}$ & 7 & 4 & & & & & & & & 11 & $\begin{array}{r}0,0 \\
5\end{array}$ \\
\hline Asteraceae & Tagetes multiflora Kunth & $\begin{array}{l}\text { Yakutsintsu, } \\
\text { chinchu, } \\
\text { tsintsu, tuna } \\
\text { tsintsu }\end{array}$ & 10 & 5 & & 29 & 1 & 19 & & & & 64 & $\begin{array}{r}0,3 \\
2\end{array}$ \\
\hline Asteraceae & Tanacetum parthenium (L.) Sch.Bip. & $\begin{array}{l}\text { María wayta, } \\
\text { santa María }\end{array}$ & 11 & & & & & & 2 & & & 13 & $\begin{array}{r}0,0 \\
6\end{array}$ \\
\hline Asteraceae & Taraxacum officinale F.H. Wigg. & $\begin{array}{l}\text { Achikuria, } \\
\text { chikuria }\end{array}$ & 32 & 3 & & & & & & & & 35 & $\begin{array}{r}0,1 \\
7\end{array}$ \\
\hline Asteraceae & Viguiera lanceolata Britton & $\begin{array}{l}\text { Sunchu, latak } \\
\text { suncho, } \\
\text { sunchus, piñaa }\end{array}$ & 2 & 24 & & & & & & & & 26 & $\begin{array}{r}0,1 \\
3\end{array}$ \\
\hline Asteraceae & Werneria nubigena Kunth & Wiskur cibulla & 1 & & & & & 2 & & & & 3 & $\begin{array}{r}0,0 \\
1\end{array}$ \\
\hline Asteraceae & Xanthium spinosum L. & Juan Alonso & 15 & & & & & & & & & 15 & $\begin{array}{r}0,0 \\
7\end{array}$ \\
\hline Basellaceae & Anredera diffusa (Moq.) Sperling & $\begin{array}{l}\text { Atojpaullucun, } \\
\text { tuna ullucu }\end{array}$ & 5 & 4 & & & & & & & & 9 & $\begin{array}{r}0,0 \\
4\end{array}$ \\
\hline Basellaceae & Ullucus tuberosus Caldas & Tuna ullucu & 4 & 3 & & & & 1 & & & & 8 & $\begin{array}{r}0,0 \\
4\end{array}$ \\
\hline $\begin{array}{l}\text { Berberidace } \\
\text { ae }\end{array}$ & Berberis carinata Lechl. & Estrella keru & & & 2 & & & & & & & 2 & $\begin{array}{r}0,0 \\
1\end{array}$ \\
\hline $\begin{array}{l}\text { Berberidace } \\
\text { ae }\end{array}$ & Berberis lutea Ruiz \& Pav. & $\begin{array}{l}\text { Estrella keru, } \\
\text { estrella kasha, } \\
\text { shekiash }\end{array}$ & & & 10 & & 9 & & & & & 19 & $\begin{array}{r}0,0 \\
9\end{array}$ \\
\hline Betulaceae & Alnus acuminata Kunth & Ramrash, aliso & 20 & 5 & 45 & & 40 & & 7 & & & $\begin{array}{r}11 \\
7\end{array}$ & $\begin{array}{r}0,5 \\
8\end{array}$ \\
\hline $\begin{array}{l}\text { Bignoniace } \\
\text { ae }\end{array}$ & Delostoma dentatum D.Don & Kara, kantu & & & 3 & & & & & & & 3 & $\begin{array}{r}0,0 \\
1\end{array}$ \\
\hline $\begin{array}{l}\text { Bignoniace } \\
\text { ae }\end{array}$ & $\begin{array}{l}\text { Tecoma stans var. sambucifolia (Kunth) } \\
\text { J.R.I.Wood }\end{array}$ & Huaroma & & & 10 & & 14 & & & & & 24 & $\begin{array}{r}0,1 \\
2\end{array}$ \\
\hline $\begin{array}{l}\text { Boraginace } \\
\text { ae }\end{array}$ & Cryptantha sp. (R. Castañeda 737) & $\begin{array}{l}\text { Mishishinua, } \\
\text { mishikasha }\end{array}$ & 1 & 7 & & & & & & & & 8 & $\begin{array}{r}0,0 \\
4\end{array}$ \\
\hline $\begin{array}{l}\text { Boraginace } \\
\text { ae }\end{array}$ & Heliotropium angiospermum Murray & Cola de alacrán & 1 & & & & & & & & & 1 & $\begin{array}{r}0,0 \\
0\end{array}$ \\
\hline $\begin{array}{l}\text { Boraginace } \\
\text { ae }\end{array}$ & Heliotropium incanum Ruiz \& Pav. & Azularia & & 7 & 7 & & & & 4 & & & 18 & $\begin{array}{r}0,0 \\
9\end{array}$ \\
\hline $\begin{array}{l}\text { Boraginace } \\
\text { ae }\end{array}$ & Phacelia secunda J.F.Gmel. & Mishikasha & & 3 & & & & & & & & 3 & $\begin{array}{r}0,0 \\
1\end{array}$ \\
\hline $\begin{array}{l}\text { Boraginace } \\
\text { ae }\end{array}$ & Wigandia urens (Ruiz \& Pav.) Kunth & Puma shinua & 9 & & & & & & & & & 9 & $\begin{array}{r}0,0 \\
4\end{array}$ \\
\hline $\begin{array}{l}\text { Brassicacea } \\
\text { e }\end{array}$ & Brassica rapa L. & $\begin{array}{l}\text { Ñapus, hitqa, } \\
\text { tuktu, ataqo, } \\
\text { mostaza, colis }\end{array}$ & 14 & 17 & & 42 & & & & & & 73 & $\begin{array}{r}0,3 \\
6\end{array}$ \\
\hline $\begin{array}{l}\text { Brassicacea } \\
\text { e }\end{array}$ & Capsella bursa-pastoris (L.) Medik. & $\begin{array}{l}\text { Shullak kiwa, } \\
\text { shullak qura, } \\
\text { yullaq wayta }\end{array}$ & 1 & 5 & & & & & & 6 & & 12 & $\begin{array}{r}0,0 \\
6\end{array}$ \\
\hline $\begin{array}{l}\text { Brassicacea } \\
\text { e }\end{array}$ & Descurainia myriophylla (Willd.) R.E.Fr. & Hakapashillun & & 3 & & & & & & 1 & & 4 & $\begin{array}{r}0,0 \\
2\end{array}$ \\
\hline
\end{tabular}


Apéndice 2. Lista de la flora silvestre útil del distrito de Pamparomás, Ancash. MED = Medicinal, APA = Alimento para animales, $\mathrm{COM}=$ Combustible, $\mathrm{ALI}=$ Alimenticio, $\mathrm{MAT}=$ Materiales, $\mathrm{SOC}=$ Social, $\mathrm{AMB}=\mathrm{Ambiental}, \mathrm{TOX}=$ Tóxica, ETN = Etnoveterinario, RU = Reportes de uso, IC = Importancia Cultural.

\begin{tabular}{|c|c|c|c|c|c|c|c|c|c|c|c|c|c|}
\hline Familia & Especie & $\begin{array}{l}\text { Nombre } \\
\text { vernáculo }\end{array}$ & $\begin{array}{l}\text { M } \\
\text { E } \\
\text { D }\end{array}$ & $\begin{array}{l}\text { AP } \\
\text { A }\end{array}$ & $\begin{array}{l}\mathrm{CO} \\
\mathrm{M}\end{array}$ & $\begin{array}{l}\text { A } \\
\text { LI }\end{array}$ & $\begin{array}{l}\text { M } \\
\text { AT }\end{array}$ & $\begin{array}{l}\mathrm{SO} \\
\mathrm{C}\end{array}$ & $\begin{array}{l}\mathrm{AM} \\
\mathrm{B}\end{array}$ & $\begin{array}{l}\text { TO } \\
\mathrm{X}\end{array}$ & $\begin{array}{l}\text { ET } \\
N\end{array}$ & RU & IC \\
\hline $\begin{array}{l}\text { Brassicacea } \\
\text { e }\end{array}$ & Lepidium bipinnatifidum Desv. & Chichikraa & & & & & & & & 3 & & 3 & $\begin{array}{r}0,0 \\
1\end{array}$ \\
\hline $\begin{array}{l}\text { Brassicacea } \\
\text { e }\end{array}$ & Lepidium chichicara Desv. & Chichikraa & 13 & 1 & & & & & & 6 & & 20 & $\begin{array}{r}0,1 \\
0\end{array}$ \\
\hline $\begin{array}{l}\text { Brassicacea } \\
\text { e }\end{array}$ & Rorippa nasturtium-aquaticum (L.) Hayek & $\begin{array}{l}\text { Yuraq birrus, } \\
\text { berros blanco }\end{array}$ & 33 & 7 & & 42 & & & & & & 82 & $\begin{array}{r}0,4 \\
1\end{array}$ \\
\hline $\begin{array}{l}\text { Bromeliace } \\
\text { ae }\end{array}$ & Puya sp. (R. Castañeda 631) & Keski & & 6 & 3 & 2 & 1 & 1 & & & & 13 & $\begin{array}{r}0,0 \\
6\end{array}$ \\
\hline $\begin{array}{l}\text { Bromeliace } \\
\text { ae }\end{array}$ & Tillandsia humilis C.Presl & Wiqlla & & 8 & & 5 & & & 3 & & & 16 & $\begin{array}{r}0,0 \\
8\end{array}$ \\
\hline Cactaceae & $\begin{array}{l}\text { Austrocylindropuntia floccosa (Salm-Dyck } \\
\text { ex Winterfeld) F.Ritter }\end{array}$ & Warkukasha & & 2 & & 4 & & & & & & 6 & $\begin{array}{r}0,0 \\
3\end{array}$ \\
\hline Cactaceae & $\begin{array}{l}\text { Austrocylindropuntia subulata } \\
\text { (Muehlenpf.) Backeb. }\end{array}$ & $\begin{array}{l}\text { Kasha, } \\
\text { unkushkasha }\end{array}$ & 5 & 2 & 1 & & & & & & & 8 & $\begin{array}{r}0,0 \\
4\end{array}$ \\
\hline Cactaceae & $\begin{array}{l}\text { Cleistocactus fieldianus (Britton \& Rose) } \\
\text { D.R.Hunt }\end{array}$ & $\begin{array}{l}\text { Shankee, } \\
\text { shankee kasha, } \\
\text { lapsop }\end{array}$ & 5 & & & 15 & & & & & & 20 & $\begin{array}{r}0,1 \\
0\end{array}$ \\
\hline Cactaceae & Corryocactus brachycladus F.Ritter & Shurukasha & 7 & 2 & & 9 & & & & & & 18 & $\begin{array}{r}0,0 \\
9\end{array}$ \\
\hline Cactaceae & Loxanthocereus sulcifer Rauh \& Backeb. & $\begin{array}{l}\text { Allqupa } \\
\text { shankeenin, } \\
\text { shankee de } \\
\text { perro }\end{array}$ & 3 & & & 9 & & & & & & 12 & $\begin{array}{r}0,0 \\
6\end{array}$ \\
\hline Cactaceae & $\begin{array}{l}\text { Matucana haynei (Otto ex Salm-Dyck) } \\
\text { Britton \& Rose }\end{array}$ & Kurikasha & 8 & 2 & & 6 & 1 & & & & & 17 & $\begin{array}{r}0,0 \\
8\end{array}$ \\
\hline Cactaceae & Opuntia ficus-indica (L.) Mill. & Tuna, tunas & 8 & & & 50 & & & & & & 58 & $\begin{array}{r}0,2 \\
9\end{array}$ \\
\hline $\begin{array}{l}\text { Calceolaria } \\
\text { ceae }\end{array}$ & Calceolaria angustiflora Ruiz \& Pav. & $\begin{array}{l}\text { Urkupashta, } \\
\text { hierba de } \\
\text { culebra, } \\
\text { karwashlaria }\end{array}$ & 1 & & & & & & & & & 1 & $\begin{array}{r}0,0 \\
0\end{array}$ \\
\hline $\begin{array}{l}\text { Calceolaria } \\
\text { ceae }\end{array}$ & Calceolaria chelidonioides Kunth & $\begin{array}{lr}\text { Bolsa } & \text { del } \\
\text { pastor, arhuee } \\
\text { Tullu }\end{array}$ & 1 & & & & & & & & & 1 & $\begin{array}{r}0,0 \\
0\end{array}$ \\
\hline $\begin{array}{l}\text { Calceolaria } \\
\text { ceae }\end{array}$ & Calceolaria pinnata L. & $\begin{array}{l}\text { Urkupashta, } \\
\text { bolsa del } \\
\text { pastor, arhuee } \\
\text { tullu }\end{array}$ & 1 & & & & & & & & & 1 & $\begin{array}{r}0,0 \\
0\end{array}$ \\
\hline $\begin{array}{l}\text { Calceolaria } \\
\text { ceae }\end{array}$ & Calceolaria rugulosa Edwin & $\begin{array}{l}\text { Urkupashta, } \\
\text { pashtak, lariaq } \\
\text { qura }\end{array}$ & 8 & 4 & 2 & & & 5 & & & & 19 & $\begin{array}{r}0,0 \\
9\end{array}$ \\
\hline $\begin{array}{l}\text { Calceolaria } \\
\text { ceae }\end{array}$ & $\begin{array}{l}\text { Calceolaria utricularioides Hook. ex } \\
\text { Benth. }\end{array}$ & $\begin{array}{l}\text { Arhuee tullu, } \\
\text { arhuee tulli }\end{array}$ & 11 & & & & & & & & & 11 & $\begin{array}{r}0,0 \\
5\end{array}$ \\
\hline $\begin{array}{l}\text { Campanula } \\
\text { ceae }\end{array}$ & Lobelia decurrens Cav. & Kuntoya & & 2 & & & & & & & 8 & 10 & $\begin{array}{r}0,0 \\
5\end{array}$ \\
\hline $\begin{array}{l}\text { Capparacea } \\
\text { e }\end{array}$ & Colicodendron scabridum (Kunth) Seem. & Sapote & & & 2 & & & & & & & 2 & $\begin{array}{r}0,0 \\
1\end{array}$ \\
\hline $\begin{array}{l}\text { Caprifoliac } \\
\text { eae }\end{array}$ & Dipsacus fullonum L. & Cardón & & & & & 3 & & & & & 3 & $\begin{array}{r}0,0 \\
1\end{array}$ \\
\hline Caricaceae & Carica candicans A.Gray & Kemis, mito & 8 & & & 13 & & & & & & 21 & $\begin{array}{r}0,1 \\
0\end{array}$ \\
\hline Caricaceae & Carica parviflora (A.DC.) Solms & Huanarpo & 2 & & & & & & & & & 2 & $\begin{array}{r}0,0 \\
1\end{array}$ \\
\hline $\begin{array}{l}\text { Caryophyll } \\
\text { aceae }\end{array}$ & Stellaria cuspidata Willd. ex Schltdl. & $\begin{array}{l}\text { Tuullush, } \\
\text { qopikqora }\end{array}$ & 17 & 5 & & & & & & & & 22 & $\begin{array}{r}0,1 \\
1\end{array}$ \\
\hline $\begin{array}{l}\text { Crassulacea } \\
\text { e }\end{array}$ & Crassula connata (Ruiz \& Pav.) A.Berger & $\begin{array}{l}\text { Quinhuanya, } \\
\text { quiñuanya, } \\
\text { kashpa kashpa }\end{array}$ & 5 & & & & & 2 & & & & 7 & $\begin{array}{r}0,0 \\
3\end{array}$ \\
\hline Cucurbitace & Sicyos baderoa Hook. \& Arn. & Shapash qura & & 9 & & & 15 & & & & & 24 & 0,1 \\
\hline
\end{tabular}


Apéndice 2. Lista de la flora silvestre útil del distrito de Pamparomás, Ancash. MED = Medicinal, APA = Alimento para animales, $\mathrm{COM}=$ Combustible, $\mathrm{ALI}=$ Alimenticio, $\mathrm{MAT}=$ Materiales, $\mathrm{SOC}=$ Social, $\mathrm{AMB}=\mathrm{Ambiental}, \mathrm{TOX}=$ Tóxica, ETN = Etnoveterinario, RU = Reportes de uso, IC = Importancia Cultural.

\begin{tabular}{|c|c|c|c|c|c|c|c|c|c|c|c|c|c|}
\hline Familia & Especie & $\begin{array}{l}\text { Nombre } \\
\text { vernáculo }\end{array}$ & $\begin{array}{l}\text { M } \\
\text { E } \\
\text { D }\end{array}$ & $\begin{array}{l}\mathrm{AP} \\
\mathrm{A}\end{array}$ & $\begin{array}{l}\mathrm{CO} \\
\mathrm{M}\end{array}$ & $\begin{array}{l}\text { A } \\
\text { LI }\end{array}$ & $\begin{array}{l}\text { M } \\
\text { AT }\end{array}$ & $\begin{array}{l}\text { SO } \\
\text { C }\end{array}$ & $\begin{array}{l}\mathrm{AM} \\
\mathrm{B}\end{array}$ & $\begin{array}{l}\mathrm{TO} \\
\mathrm{X}\end{array}$ & $\begin{array}{l}\text { ET } \\
N\end{array}$ & RU & IC \\
\hline ae & & & & & & & & & & & & & 2 \\
\hline Cyperaceae & Carex hebetata Boott & Raqraq & 1 & 4 & & & 5 & & & & & 10 & 0,0 \\
\hline $\begin{array}{l}\text { Ephedracea } \\
\text { e }\end{array}$ & $\begin{array}{l}\text { Ephedra americana Humb. \& Bonpl. ex } \\
\text { Willd. }\end{array}$ & $\begin{array}{l}\text { Pinco pinco, } \\
\text { wakwa }\end{array}$ & 2 & & & & & & & & & 2 & $\begin{array}{r}0,0 \\
1\end{array}$ \\
\hline $\begin{array}{l}\text { Ephedracea } \\
\text { e }\end{array}$ & Ephedra rupestris Benth. & $\begin{array}{l}\text { Wakwa, irka } \\
\text { wakwa, } \\
\text { yuquispa } \\
\text { umllan }\end{array}$ & 5 & & & 6 & & & & & & 11 & $\begin{array}{r}0,0 \\
5\end{array}$ \\
\hline $\begin{array}{l}\text { Equisetacea } \\
\mathrm{e}\end{array}$ & Equisetum bogotense Kunth & $\begin{array}{l}\text { Shaka, cola de } \\
\text { caballo }\end{array}$ & 39 & 14 & & 8 & 1 & & & & & 62 & $\begin{array}{r}0,3 \\
1\end{array}$ \\
\hline $\begin{array}{l}\text { Escalloniac } \\
\text { eae }\end{array}$ & Escallonia pendula (Ruiz \& Pav.) Pers. & Chiri & & & 2 & & 1 & & & & & 3 & $\begin{array}{r}0,0 \\
1\end{array}$ \\
\hline $\begin{array}{l}\text { Euphorbiac } \\
\text { eae }\end{array}$ & Croton ruizianus Müll.Arg. & $\begin{array}{l}\text { Kasmanyaa, } \\
\text { Kasmenyee }\end{array}$ & 11 & & 8 & & & & & & & 19 & $\begin{array}{r}0,0 \\
9\end{array}$ \\
\hline $\begin{array}{l}\text { Euphorbiac } \\
\text { eae }\end{array}$ & $\begin{array}{l}\text { Euphorbia huanchahana (Klotzsch \& } \\
\text { Garcke) Boiss. }\end{array}$ & $\begin{array}{l}\text { Mishuaka, } \\
\text { mishuania }\end{array}$ & 3 & & & & & & & 2 & & 5 & $\begin{array}{r}0,0 \\
2\end{array}$ \\
\hline $\begin{array}{l}\text { Euphorbiac } \\
\text { eae }\end{array}$ & Jatropha macrantha Müll.Arg. & Nuntu & & & 4 & & & & & 5 & & 9 & $\begin{array}{r}0,0 \\
4\end{array}$ \\
\hline $\begin{array}{l}\text { Euphorbiac } \\
\text { eae }\end{array}$ & Ricinus communis L. & Aceite qora & 6 & & 1 & & & & & & & 7 & $\begin{array}{r}0,0 \\
3\end{array}$ \\
\hline $\begin{array}{l}\text { Euphorbiac } \\
\text { eae }\end{array}$ & Sebastiania obtusifolia Pax \& K.Hoffm. & $\begin{array}{l}\text { Hueqesh, } \\
\text { hueqish }\end{array}$ & & 5 & 9 & & 2 & 1 & & & & 17 & $\begin{array}{r}0,0 \\
8\end{array}$ \\
\hline Fabaceae & Acacia macracantha Willd. & No reportado & & & & & 2 & & & & & 2 & $\begin{array}{r}0,0 \\
1\end{array}$ \\
\hline Fabaceae & Astragalus garbancillo Cav. & Patsapa makin & & & & & & 4 & & & & 4 & $\begin{array}{r}0,0 \\
2\end{array}$ \\
\hline Fabaceae & Caesalpinia decapetala (Roth) Alston & Uña de gato & & & & & & & 5 & & & 5 & $\begin{array}{r}0,0 \\
2\end{array}$ \\
\hline Fabaceae & Caesalpinia spinosa (Molina) Kuntze & Karanka, tara & 16 & & 12 & 13 & 11 & & & & & 52 & $\begin{array}{r}0,2 \\
6\end{array}$ \\
\hline Fabaceae & Dalea cylindrica Hook. & $\begin{array}{l}\text { Intipawarmin, } \\
\text { intipanawin, } \\
\text { inti qura }\end{array}$ & & 11 & & & 5 & 10 & & & & 26 & $\begin{array}{r}0,1 \\
3\end{array}$ \\
\hline Fabaceae & Desmodium intortum (Mill.) Urb. & Pega pega & & 11 & & & & & & & & 11 & $\begin{array}{r}0,0 \\
5\end{array}$ \\
\hline Fabaceae & Desmodium molliculum (Kunth) DC. & $\begin{array}{l}\text { Allqupachaqui } \\
\text { n, pati perro }\end{array}$ & & 8 & & & & & & & & 8 & $\begin{array}{r}0,0 \\
4\end{array}$ \\
\hline Fabaceae & Lupinus condensiflorus C.P.Sm. & Taaya & & 7 & 18 & & & & 3 & & & 28 & $\begin{array}{r}0,1 \\
4\end{array}$ \\
\hline Fabaceae & Lupinus lindleyanus J.Agardh & $\begin{array}{l}\text { Taullish, tuna, } \\
\text { tauri, } \\
\text { ircataullish }\end{array}$ & & 3 & 10 & & & & & & & 13 & $\begin{array}{r}0,0 \\
6\end{array}$ \\
\hline Fabaceae & Medicago polymorpha L. & $\begin{array}{l}\text { Tríbul, trébol } \\
\text { hembra }\end{array}$ & 2 & 33 & & & & & & & & 35 & $\begin{array}{r}0,1 \\
7\end{array}$ \\
\hline Fabaceae & Melilotus indicus (L.) All. & $\begin{array}{l}\text { Alfar macho, } \\
\text { trébol macho }\end{array}$ & & 9 & & & & & & & & 9 & $\begin{array}{r}0,0 \\
4\end{array}$ \\
\hline Fabaceae & Otholobium pubescens (Poir.) J.W. Grimes & Kulen, kulin & 17 & & & & & & & & & 17 & $\begin{array}{r}0,0 \\
8\end{array}$ \\
\hline Fabaceae & $\begin{array}{l}\text { Senna versicolor (Vogel) H.S.Irwin \& } \\
\text { Barneby }\end{array}$ & Huishllak & & & & & 3 & & & & & 3 & $\begin{array}{r}0,0 \\
1\end{array}$ \\
\hline Fabaceae & Spartium junceum L. & Retama & 13 & 6 & 1 & & & 8 & 16 & & & 44 & $\begin{array}{r}0,2 \\
2\end{array}$ \\
\hline Fabaceae & Trifolium amabile Kunth & Ankuyá & 1 & & & & & & & & & 1 & $\begin{array}{r}0,0 \\
0\end{array}$ \\
\hline Fabaceae & Vicia andicola Kunth & $\begin{array}{l}\text { Haka mirasiq, } \\
\text { shintu, mullu } \\
\text { mullu }\end{array}$ & & 17 & & 1 & & 8 & & & & 26 & $\begin{array}{r}0,1 \\
3\end{array}$ \\
\hline
\end{tabular}


Apéndice 2. Lista de la flora silvestre útil del distrito de Pamparomás, Ancash. MED = Medicinal, APA = Alimento para animales, $\mathrm{COM}=$ Combustible, $\mathrm{ALI}=$ Alimenticio, $\mathrm{MAT}=$ Materiales, $\mathrm{SOC}=$ Social, $\mathrm{AMB}=\mathrm{Ambiental}, \mathrm{TOX}=$ Tóxica, ETN = Etnoveterinario, RU = Reportes de uso, IC = Importancia Cultural.

\begin{tabular}{|c|c|c|c|c|c|c|c|c|c|c|c|c|c|}
\hline Familia & Especie & $\begin{array}{l}\text { Nombre } \\
\text { vernáculo }\end{array}$ & $\begin{array}{l}\text { M } \\
\text { E } \\
\text { D }\end{array}$ & $\begin{array}{l}\text { AP } \\
\text { A }\end{array}$ & $\begin{array}{l}\mathrm{CO} \\
\mathrm{M}\end{array}$ & $\begin{array}{l}\text { A } \\
\text { LI }\end{array}$ & $\begin{array}{l}\text { M } \\
\text { AT }\end{array}$ & $\begin{array}{l}\mathrm{SO} \\
\mathrm{C}\end{array}$ & $\begin{array}{l}\mathrm{AM} \\
\mathrm{B}\end{array}$ & $\begin{array}{l}\text { TO } \\
\mathrm{X}\end{array}$ & $\begin{array}{l}\text { ET } \\
N\end{array}$ & RU & IC \\
\hline $\begin{array}{l}\text { Geraniacea } \\
\text { e }\end{array}$ & Erodium malacoides (L.) L'Hér. & $\begin{array}{l}\text { Alfiler, } \\
\text { alfilerillo, } \\
\text { timpicha }\end{array}$ & 13 & 22 & & 1 & & & & & & 36 & $\begin{array}{r}0,1 \\
8\end{array}$ \\
\hline $\begin{array}{l}\text { Grossularia } \\
\text { ceae }\end{array}$ & Ribes viscosum Cels ex Dum.Cours. & Arranpushko & & & & 1 & & & & & & 1 & $\begin{array}{r}0,0 \\
0\end{array}$ \\
\hline $\begin{array}{l}\text { Juglandace } \\
\text { a }\end{array}$ & Juglans neotropica Diels & Nogal & 15 & & 18 & 18 & 33 & & & & & 84 & $\begin{array}{r}0,4 \\
2\end{array}$ \\
\hline $\begin{array}{l}\text { Krameriace } \\
\text { ae }\end{array}$ & $\begin{array}{l}\text { Krameria lappacea (Dombey) Burdet \& } \\
\text { B.B. Simpson }\end{array}$ & No reportado & & 1 & & & & & & & & 1 & $\begin{array}{r}0,0 \\
0\end{array}$ \\
\hline Lamiaceae & $\begin{array}{l}\text { Clinopodium sericeum (C. Presl ex Benth.) } \\
\text { Govaerts }\end{array}$ & Koyal & 1 & & & & & & & & & 1 & $\begin{array}{r}0,0 \\
0\end{array}$ \\
\hline Lamiaceae & Minthostachys mollis (Benth.) Griseb. & Munqa, muña & 70 & 11 & 1 & 7 & & & & & & 89 & $\begin{array}{r}0,4 \\
4\end{array}$ \\
\hline Lamiaceae & Salvia oppositiflora Ruiz \& Pav. & $\begin{array}{l}\text { Shokumpa } \\
\text { wayta }\end{array}$ & 1 & & & & & 12 & & & & 13 & $\begin{array}{r}0,0 \\
6\end{array}$ \\
\hline Lamiaceae & Salvia sagittata Ruiz \& Pav. & Azul & & & & & 3 & & & & & 3 & $\begin{array}{r}0,0 \\
1\end{array}$ \\
\hline Lamiaceae & Salvia speciosa C.Presl ex Benth. & $\begin{array}{l}\text { Pinhuakshu, } \\
\text { pinkush, } \\
\text { shukumunti }\end{array}$ & 6 & 10 & 11 & & & & & & & 27 & $\begin{array}{r}0,1 \\
3\end{array}$ \\
\hline Loasaceae & Caiophora cirsiifolia C.Presl & $\begin{array}{l}\text { Latak shinua, } \\
\text { gachgu shinua, } \\
\text { shishu shinua }\end{array}$ & 13 & & & & & 1 & & & & 14 & $\begin{array}{r}0,0 \\
7\end{array}$ \\
\hline Loasaceae & Mentzelia scabra Kunth & $\begin{array}{l}\text { Anguaratee, } \\
\text { pega pega, tapa } \\
\text { tapa }\end{array}$ & 22 & & & & & & & & & 22 & $\begin{array}{r}0,1 \\
1\end{array}$ \\
\hline Loasaceae & Nasa grandiflora (Desr.) Weigend & $\begin{array}{l}\text { Boton shinua, } \\
\text { hatun shinua, } \\
\text { puma shinua, } \\
\text { urqu shinua }\end{array}$ & 4 & & & 5 & & & & & & 9 & $\begin{array}{r}0,0 \\
4\end{array}$ \\
\hline $\begin{array}{l}\text { Loranthace } \\
\text { ae }\end{array}$ & Tristerix peruvianus (Pacz.) Kuijt & $\begin{array}{l}\text { Pupa, suelda } \\
\text { con suelda }\end{array}$ & 5 & & & & & & & & 3 & 8 & $\begin{array}{r}0,0 \\
4\end{array}$ \\
\hline Malvaceae & Eriotheca ruizii (K.Schum.) A.Robyns & Utko, aceqora & & & 2 & & 1 & & & & & 3 & $\begin{array}{r}0,0 \\
1\end{array}$ \\
\hline Malvaceae & $\begin{array}{l}\text { Fuertesimalva leptocalyx } \quad \text { (Krapov.) } \\
\text { Fryxell }\end{array}$ & Puchmus & & 13 & & & & & & & & 13 & $\begin{array}{r}0,0 \\
6\end{array}$ \\
\hline Malvaceae & Lepechinia meyenii (Walp.) Epling & Salvia & 14 & & & & & 1 & & & & 15 & $\begin{array}{r}0,0 \\
7\end{array}$ \\
\hline Malvaceae & Malva parviflora $\mathrm{L}$. & Malvas & 15 & 15 & & 1 & & & & & & 31 & $\begin{array}{r}0,1 \\
5\end{array}$ \\
\hline Malvaceae & Sida rhombifolia L. & $\begin{array}{l}\text { Ankuyá, } \\
\text { anguya }\end{array}$ & 3 & & & & 4 & & & & & 7 & $\begin{array}{r}0,0 \\
3\end{array}$ \\
\hline Moraceae & Ficus trigona L.f. & Pati & 13 & 4 & 17 & & & & & & 4 & 38 & $\begin{array}{r}0,1 \\
9\end{array}$ \\
\hline Myrtaceae & $\begin{array}{l}\text { Myrcianthes quinqueloba (McVaugh) } \\
\text { McVaugh }\end{array}$ & Karapacho & & & & & 5 & & & & & 5 & $\begin{array}{r}0,0 \\
2\end{array}$ \\
\hline $\begin{array}{l}\text { Nyctaginac } \\
\text { eae }\end{array}$ & Mirabilis campanulata Heimerl & $\begin{array}{l}\text { Natikshu, } \\
\text { ñatikshu }\end{array}$ & & 5 & & & & & & & & 5 & $\begin{array}{r}0,0 \\
2\end{array}$ \\
\hline Onagraceae & Oenothera laciniata Hill & $\begin{array}{l}\text { Ticlanaria, } \\
\text { licshanya }\end{array}$ & 2 & 2 & & & & & & & 6 & 10 & $\begin{array}{r}0,0 \\
5\end{array}$ \\
\hline Onagraceae & Oenothera rosea L'Hér. ex Aiton & $\begin{array}{l}\text { Santa Lucía, } \\
\text { chupa sangre }\end{array}$ & 18 & & & & & & & & & 18 & $\begin{array}{r}0,0 \\
9\end{array}$ \\
\hline $\begin{array}{l}\text { Orchidacea } \\
\text { e }\end{array}$ & Porphyrostachys pilifera (Kunth) Rchb.f. & Hueqlla & & & & & & & 3 & & & 3 & $\begin{array}{r}0,0 \\
1\end{array}$ \\
\hline $\begin{array}{l}\text { Oxalidacea } \\
\text { e }\end{array}$ & Oxalis megalorrhiza Jacq. & $\begin{array}{l}\text { Puchgus, } \\
\text { ucaullo, } \\
\text { arashpayucan }\end{array}$ & 4 & 6 & & 21 & 1 & & & & & 32 & $\begin{array}{r}0,1 \\
6\end{array}$ \\
\hline $\begin{array}{l}\text { Papaverace } \\
\text { ae }\end{array}$ & Argemone mexicana L. & $\begin{array}{l}\text { San Carlos, } \\
\text { cardo santo }\end{array}$ & 11 & & & & & & & & 4 & 15 & $\begin{array}{r}0,0 \\
7\end{array}$ \\
\hline
\end{tabular}


Apéndice 2. Lista de la flora silvestre útil del distrito de Pamparomás, Ancash. MED = Medicinal, APA = Alimento para animales, $\mathrm{COM}=$ Combustible, $\mathrm{ALI}=$ Alimenticio, $\mathrm{MAT}=$ Materiales, $\mathrm{SOC}=$ Social, $\mathrm{AMB}=\mathrm{Ambiental}, \mathrm{TOX}=$ Tóxica, ETN = Etnoveterinario, RU = Reportes de uso, IC = Importancia Cultural.

\begin{tabular}{|c|c|c|c|c|c|c|c|c|c|c|c|c|c|}
\hline Familia & Especie & $\begin{array}{l}\text { Nombre } \\
\text { vernáculo }\end{array}$ & $\begin{array}{l}\text { M } \\
\mathrm{E} \\
\mathrm{D}\end{array}$ & $\begin{array}{l}\text { AP } \\
\text { A }\end{array}$ & $\begin{array}{l}\mathrm{CO} \\
\mathrm{M}\end{array}$ & $\begin{array}{l}\text { A } \\
\text { LI }\end{array}$ & $\begin{array}{l}\text { M } \\
\text { AT }\end{array}$ & $\begin{array}{l}\text { SO } \\
\text { C }\end{array}$ & $\begin{array}{l}\mathrm{AM} \\
\mathrm{B}\end{array}$ & $\begin{array}{l}\text { TO } \\
\mathrm{X}\end{array}$ & $\begin{array}{l}\text { ET } \\
N\end{array}$ & RU & IC \\
\hline $\begin{array}{l}\text { Passiflorace } \\
\text { ae }\end{array}$ & Passiflora peduncularis Cav. & $\begin{array}{l}\text { Purush, tuna } \\
\text { purush }\end{array}$ & & 8 & & 14 & & & & 1 & & 23 & $\begin{array}{r}0,1 \\
1\end{array}$ \\
\hline $\begin{array}{l}\text { Passiflorace } \\
\text { ae }\end{array}$ & Passiflora tripartita (Juss.) Poir. & $\begin{array}{l}\text { Purush, purush } \\
\text { redondo }\end{array}$ & & & & 2 & & & & & & 2 & $\begin{array}{r}0,0 \\
1\end{array}$ \\
\hline $\begin{array}{l}\text { Phrymacea } \\
\text { e }\end{array}$ & Mimulus glabratus Kunth & $\begin{array}{l}\text { Karuash birrus, } \\
\text { berros amarillo, } \\
\text { berro dulce }\end{array}$ & 19 & & & & & & & & & 19 & $\begin{array}{r}0,0 \\
9\end{array}$ \\
\hline Piperaceae & $\begin{array}{l}\text { Peperomia inaequalifolia var. galioides } \\
\text { (Kunth) Pino }\end{array}$ & $\begin{array}{l}\text { Congona, } \\
\text { tunacongona, } \\
\text { congona } \\
\text { silvestre }\end{array}$ & 28 & & & & & & & & & 28 & $\begin{array}{r}0,1 \\
4\end{array}$ \\
\hline Piperaceae & Piper barbatum Kunth & $\begin{array}{l}\text { Huasgatoo, } \\
\text { matico }\end{array}$ & 6 & & & & & & & & & 6 & $\begin{array}{r}0,0 \\
3\end{array}$ \\
\hline $\begin{array}{l}\text { Plantaginac } \\
\text { eae }\end{array}$ & Plantago lanceolata L. & $\begin{array}{l}\text { Llantén, llantén } \\
\text { macho }\end{array}$ & 52 & 3 & & 4 & & & & & & 59 & $\begin{array}{r}0,2 \\
9\end{array}$ \\
\hline $\begin{array}{l}\text { Plantaginac } \\
\text { eae }\end{array}$ & Plantago major L. & Llantén & 1 & & & & & & & & & 1 & $\begin{array}{r}0,0 \\
0\end{array}$ \\
\hline $\begin{array}{l}\text { Plantaginac } \\
\text { eae }\end{array}$ & Scoparia dulcis L. & $\begin{array}{l}\text { Hierba del } \\
\text { susto }\end{array}$ & 1 & & & & & & & & & 1 & $\begin{array}{r}0,0 \\
0\end{array}$ \\
\hline $\begin{array}{l}\text { Plantaginac } \\
\text { eae }\end{array}$ & Veronica persica Poir. & Lucila & & 3 & & 1 & & & & & & 4 & $\begin{array}{r}0,0 \\
2\end{array}$ \\
\hline Poaceae & Avena sterilis L. & Cebadilla & 1 & 17 & & & & & & & & 18 & $\begin{array}{r}0,0 \\
9\end{array}$ \\
\hline Poaceae & Bothriochloa saccharoides (Sw.) Rydb. & Shuqlla & & 1 & & & & & & & & 1 & $\begin{array}{r}0,0 \\
0\end{array}$ \\
\hline Poaceae & Bromus berteroanus Colla & Paja & & 2 & & & & & & & & 2 & $\begin{array}{r}0,0 \\
1\end{array}$ \\
\hline Poaceae & Bromus catharticus Vahl & $\begin{array}{l}\text { Shuqlla, } \\
\text { allqupashuqllan }\end{array}$ & 1 & 4 & & & & & & & & 5 & $\begin{array}{r}0,0 \\
2\end{array}$ \\
\hline Poaceae & Calamagrostis amoena (Pilg.) Pilg. & Ichu, achu & & 15 & & & 12 & & & & & 27 & $\begin{array}{r}0,1 \\
3\end{array}$ \\
\hline Poaceae & Calamagrostis heterophylla (Wedd.) Pilg. & Paja & & 1 & & & & & & & & 1 & $\begin{array}{r}0,0 \\
0\end{array}$ \\
\hline Poaceae & $\begin{array}{l}\text { Cenchrus clandestinus (Hochst. ex Chiov.) } \\
\text { Morrone. }\end{array}$ & Kikuyo & & 1 & & & & & & & & 1 & $\begin{array}{r}0,0 \\
0\end{array}$ \\
\hline Poaceae & Cenchrus weberbaueri (Mez) Morrone & Saaria & & 3 & & & & & & & & 3 & $\begin{array}{r}0,0 \\
1\end{array}$ \\
\hline Poaceae & $\begin{array}{l}\text { Cortaderia jubata (Lemoine ex Carrière) } \\
\text { Stapf }\end{array}$ & Tseqtseq & 1 & 6 & & & & & & & & 7 & $\begin{array}{r}0,0 \\
3\end{array}$ \\
\hline Poaceae & Festuca hieronymi Hack. & Kgatsil & & 4 & & & 8 & & & & & 12 & $\begin{array}{r}0,0 \\
6\end{array}$ \\
\hline Poaceae & Hordeum muticum J.Presl & Shuqlla & & 15 & & & & & & & 5 & 20 & $\begin{array}{r}0,1 \\
0\end{array}$ \\
\hline Poaceae & Jarava ichu Ruiz \& Pav. & Ichu, achu & & 25 & & & 28 & & & & & 53 & $\begin{array}{r}0,2 \\
6\end{array}$ \\
\hline Poaceae & Lolium multiflorum Lam. & Balligo & & 5 & & & & & & & & 5 & $\begin{array}{r}0,0 \\
2\end{array}$ \\
\hline Poaceae & Nassella asplundii Hitchc. & Shuqlla & & 13 & & & & & & & & 13 & $\begin{array}{r}0,0 \\
6\end{array}$ \\
\hline Poaceae & Nassella mucronata (Kunth) R.W.Pohl & $\begin{array}{l}\text { Shuklla, } \\
\text { allqupashukllan }\end{array}$ & 3 & 9 & & & & & & & 3 & 15 & $\begin{array}{r}0,0 \\
7\end{array}$ \\
\hline Poaceae & Nassella pubiflora (Trin. \& Rupr.) É.Desv. & Paja & & 1 & & & & & & & & 1 & $\begin{array}{r}0,0 \\
0\end{array}$ \\
\hline Poaceae & Paspalum penicillatum Hook.f. & Arac kehua & & 8 & & & & & & & & 8 & $\begin{array}{r}0,0 \\
4\end{array}$ \\
\hline Poaceae & Poa carazensis Pilg. & Paja & & 1 & & & & & & & & 1 & $\begin{array}{r}0,0 \\
0\end{array}$ \\
\hline Poaceae & Poa glaberrima Tovar & Shuqlla & & 13 & & & & & & & & 13 & $\begin{array}{r}0,0 \\
6\end{array}$ \\
\hline
\end{tabular}


Apéndice 2. Lista de la flora silvestre útil del distrito de Pamparomás, Ancash. MED = Medicinal, APA = Alimento para animales, $\mathrm{COM}=$ Combustible, $\mathrm{ALI}=$ Alimenticio, $\mathrm{MAT}=$ Materiales, $\mathrm{SOC}=$ Social, $\mathrm{AMB}=\mathrm{Ambiental}, \mathrm{TOX}=$ Tóxica, ETN = Etnoveterinario, RU = Reportes de uso, IC = Importancia Cultural.

\begin{tabular}{|c|c|c|c|c|c|c|c|c|c|c|c|c|c|}
\hline Familia & Especie & $\begin{array}{l}\text { Nombre } \\
\text { vernáculo }\end{array}$ & $\begin{array}{l}\text { M } \\
\text { E } \\
\text { D }\end{array}$ & $\begin{array}{l}\text { AP } \\
\text { A }\end{array}$ & $\begin{array}{l}\mathrm{CO} \\
\mathrm{M}\end{array}$ & $\begin{array}{l}\text { A } \\
\text { LI }\end{array}$ & $\begin{array}{l}\text { M } \\
\text { AT }\end{array}$ & $\begin{array}{l}\mathrm{SO} \\
\mathrm{C}\end{array}$ & $\begin{array}{l}\mathrm{AM} \\
\mathrm{B}\end{array}$ & $\begin{array}{l}\mathrm{TO} \\
\mathrm{X}\end{array}$ & $\begin{array}{l}\mathrm{ET} \\
\mathrm{N}\end{array}$ & RU & IC \\
\hline Poaceae & Poa serpaiana Refulio & Paja & & 2 & & & & & & & & 2 & $\begin{array}{r}0,0 \\
1\end{array}$ \\
\hline Poaceae & Polypogon elongatus Kunth & Shuqlla & & 13 & & & & & & & & 13 & $\begin{array}{r}0,0 \\
6\end{array}$ \\
\hline Poaceae & Sporobolus indicus (L.) R.Br. & No reportado & & 1 & & & & & & & & 1 & $\begin{array}{r}0,0 \\
0\end{array}$ \\
\hline Poaceae & Vulpia bromoides (L.) Gray & Pajonal & & 8 & & & & & & & & 8 & $\begin{array}{r}0,0 \\
4\end{array}$ \\
\hline Poaceae & Vulpia myuros (L.) C.C.Gmel. & Paja & & 1 & & & & & & & & 1 & $\begin{array}{r}0,0 \\
0\end{array}$ \\
\hline $\begin{array}{l}\text { Polemoniac } \\
\text { eae }\end{array}$ & Cantua buxifolia Juss. ex Lam. & Cantu & & & & & & & 5 & & & 5 & $\begin{array}{r}0,0 \\
2\end{array}$ \\
\hline $\begin{array}{l}\text { Polygalacea } \\
\text { e }\end{array}$ & Monnina salicifolia Ruiz \& Pav. & $\begin{array}{l}\text { Yanash, } \\
\text { muchisa, } \\
\text { mushikura }\end{array}$ & 3 & 1 & 3 & 10 & 1 & & & & & 18 & $\begin{array}{r}0,0 \\
9\end{array}$ \\
\hline $\begin{array}{l}\text { Polygonace } \\
\text { ae }\end{array}$ & Muehlenbeckia tamnifolia (Kunth) Meisn. & $\begin{array}{l}\text { Aurushu, } \\
\text { aurinshu, } \\
\text { soksoma }\end{array}$ & & 13 & & & & & & & & 13 & $\begin{array}{r}0,0 \\
6\end{array}$ \\
\hline $\begin{array}{l}\text { Polygonace } \\
\text { ae }\end{array}$ & Rumex crispus L. & $\begin{array}{l}\text { Chuchuqura, } \\
\text { acelga }\end{array}$ & 22 & 4 & & 3 & & & & & & 29 & $\begin{array}{r}0,1 \\
4\end{array}$ \\
\hline $\begin{array}{l}\text { Portulacace } \\
\text { ae }\end{array}$ & Portulaca oleracea L. & Pilto & 3 & & & & & & & & & 3 & $\begin{array}{r}0,0 \\
1\end{array}$ \\
\hline $\begin{array}{l}\text { Portulacace } \\
\text { ae }\end{array}$ & Portulaca perennis R.E. Fr. & Tapa tapa & 3 & & & & & & & & & 3 & $\begin{array}{r}0,0 \\
1\end{array}$ \\
\hline Pteridaceae & Adiantum poiretii Wikstr. & $\begin{array}{l}\text { Culantrillo } \\
\text { pozo, culantro } \\
\text { pozo, } \\
\text { inkapakukan }\end{array}$ & 19 & & & 1 & & & & & & 20 & $\begin{array}{r}0,1 \\
0\end{array}$ \\
\hline Pteridaceae & Cheilanthes myriophylla Desv. & $\begin{array}{l}\text { San Juan kora, } \\
\text { inka coca }\end{array}$ & & & & & & 6 & & & & 6 & $\begin{array}{r}0,0 \\
3\end{array}$ \\
\hline Rosaceae & Acaena torilicarpa Bitter & $\begin{array}{l}\text { Shapra, puma } \\
\text { shapra, frutilla }\end{array}$ & & 5 & 5 & 9 & 1 & & & & & 20 & $\begin{array}{r}0,1 \\
0\end{array}$ \\
\hline Rosaceae & Fragaria indica Andrews & Fresa silvestre & & 3 & & 15 & & & & & & 18 & $\begin{array}{r}0,0 \\
9\end{array}$ \\
\hline Rosaceae & Margyricarpus pinnatus (Lam.) Kuntze & Perlas, pawaa & 10 & 4 & 11 & 5 & 1 & & & & & 31 & $\begin{array}{r}0,1 \\
5\end{array}$ \\
\hline Rosaceae & Polylepis racemosa Ruiz \& Pav. & $\begin{array}{l}\text { Quenua, } \\
\text { quenual, } \\
\text { quiñua }\end{array}$ & 15 & 5 & 40 & & 17 & & 15 & & & 92 & $\begin{array}{r}0,4 \\
6\end{array}$ \\
\hline Rosaceae & Rubus robustus P.J.Müll. & $\begin{array}{l}\text { Shira casha, } \\
\text { shiracsha }\end{array}$ & 7 & & 1 & 5 & & & 4 & & & 17 & $\begin{array}{r}0,0 \\
8\end{array}$ \\
\hline Rosaceae & Tetraglochin cristatum (Britton) Rothm. & Perla & & & 1 & & & & & & & 1 & $\begin{array}{r}0,0 \\
0\end{array}$ \\
\hline Rosaceae & Hesperomeles cuneata Lindl. & $\begin{array}{l}\text { Tuuskar, } \\
\text { manzana }\end{array}$ & 1 & 5 & 15 & 15 & 11 & & & & & 47 & $\begin{array}{r}0,2 \\
3\end{array}$ \\
\hline Rubiaceae & $\begin{array}{l}\text { Arcytophyllum thymifolium (Ruiz \& Pav.) } \\
\text { Standl. }\end{array}$ & $\begin{array}{l}\text { Wiscurpachaqu } \\
\text { in, wiscurpa } \\
\text { tukrum, } \\
\text { pichakpashillun } \\
\text { capchachak, } \\
\text { mallisma }\end{array}$ & 1 & 8 & 13 & & & & 6 & & & 20 & $\begin{array}{r}0,1 \\
0\end{array}$ \\
\hline $\begin{array}{l}\text { Schoepfiac } \\
\text { eae }\end{array}$ & Quinchamalium procumbens Ruiz \& Pav. & $\begin{array}{l}\text { Chinchimallí } \\
\text { hembra }\end{array}$ & 3 & & & & & 3 & & & & 6 & $\begin{array}{r}0,0 \\
3\end{array}$ \\
\hline $\begin{array}{l}\text { Scrophulari } \\
\text { aceae }\end{array}$ & Alonsoa acutifolia Ruiz \& Pav. & Milla milla & & 1 & & & & & & & & 1 & $\begin{array}{r}0,0 \\
0\end{array}$ \\
\hline $\begin{array}{l}\text { Scrophulari } \\
\text { aceae }\end{array}$ & Alonsoa meridionalis (L.f.) Kuntze & $\begin{array}{l}\text { Ricachekuq } \\
\text { wayta, } \\
\text { ricachekuna, } \\
\text { monte }\end{array}$ & & 1 & & & & 9 & 2 & 1 & 3 & 16 & $\begin{array}{r}0,0 \\
8\end{array}$ \\
\hline
\end{tabular}


Apéndice 2. Lista de la flora silvestre útil del distrito de Pamparomás, Ancash. MED = Medicinal, APA = Alimento para animales, $\mathrm{COM}=$ Combustible, $\mathrm{ALI}=$ Alimenticio, $\mathrm{MAT}=$ Materiales, $\mathrm{SOC}=$ Social, $\mathrm{AMB}=\mathrm{Ambiental}, \mathrm{TOX}=$ Tóxica, ETN = Etnoveterinario, RU = Reportes de uso, IC = Importancia Cultural.

\begin{tabular}{|c|c|c|c|c|c|c|c|c|c|c|c|c|c|}
\hline Familia & Especie & $\begin{array}{l}\text { Nombre } \\
\text { vernáculo }\end{array}$ & $\begin{array}{l}\text { M } \\
\text { E } \\
D\end{array}$ & $\begin{array}{l}\text { AP } \\
\text { A }\end{array}$ & $\begin{array}{l}\mathrm{CO} \\
\mathrm{M}\end{array}$ & $\begin{array}{l}\text { A } \\
\text { LI }\end{array}$ & $\begin{array}{l}\text { M } \\
\text { AT }\end{array}$ & $\begin{array}{l}\text { SO } \\
\text { C }\end{array}$ & $\begin{array}{l}\mathrm{AM} \\
\mathrm{B}\end{array}$ & $\begin{array}{l}\text { TO } \\
\mathrm{X}\end{array}$ & $\begin{array}{l}\mathrm{ET} \\
\mathrm{N}\end{array}$ & RU & IC \\
\hline & & monderejo & & & & & & & & & & & \\
\hline $\begin{array}{l}\text { Scrophulari } \\
\text { aceae }\end{array}$ & Buddleja coriacea Remy & Kulli, kolle & 3 & & 9 & & 4 & & 1 & & & 17 & $\begin{array}{r}0,0 \\
8\end{array}$ \\
\hline $\begin{array}{l}\text { Scrophulari } \\
\text { aceae }\end{array}$ & Buddleja incana Ruiz \& Pav. & $\begin{array}{l}\text { Quisuar, } \\
\text { quishuar }\end{array}$ & 17 & & 15 & & & & & & & 32 & $\begin{array}{r}0,1 \\
6\end{array}$ \\
\hline Solanaceae & Cestrum auriculatum L'Hér. & Hierba santa & 30 & & 5 & & 6 & & & 5 & & 46 & 0,2 \\
\hline Solanaceae & Cestrum conglomeratum Ruiz \& Pav. & $\begin{array}{l}\text { Llejlla, uran } \\
\text { llejlla }\end{array}$ & 5 & & & & & & & & & 5 & $\begin{array}{r}0,0 \\
2\end{array}$ \\
\hline Solanaceae & $\begin{array}{l}\text { Iochroma umbellatum (Ruiz \& Pav.) } \\
\text { Hunziker ex D'Arcy }\end{array}$ & $\begin{array}{l}\text { Rukii, San } \\
\text { Pablo, puya, } \\
\text { quebrolla }\end{array}$ & 30 & 1 & 29 & 1 & 12 & 20 & 1 & & & 94 & $\begin{array}{r}0,4 \\
7\end{array}$ \\
\hline Solanaceae & $\begin{array}{l}\text { Jaltomata bernardelloana S. Leiva \& } \\
\text { Mione }\end{array}$ & Lanqii & & 3 & & 16 & & 1 & & & & 20 & $\begin{array}{r}0,1 \\
0\end{array}$ \\
\hline Solanaceae & Jaltomata procumbens (Cav.) J.L.Gentry & $\begin{array}{l}\text { Ullma, } \\
\text { areumlla }\end{array}$ & & 10 & 2 & 7 & & & & & & 19 & $\begin{array}{r}0,0 \\
9\end{array}$ \\
\hline Solanaceae & Lycianthes lycioides (L.) Hassl. & $\begin{array}{l}\text { Tuuskar, } \\
\text { uchkus }\end{array}$ & 1 & 15 & 17 & 17 & 2 & 1 & 1 & 1 & & 55 & $\begin{array}{r}0,2 \\
7\end{array}$ \\
\hline Solanaceae & Nicandra physalodes (L.) Gaertn. & Arapamaman & 4 & & & & & & & 4 & & 8 & $\begin{array}{r}0,0 \\
4\end{array}$ \\
\hline Solanaceae & Nicotiana glutinosa L. & Asiaq qora & & & & & & & & & 8 & 8 & $\begin{array}{r}0,0 \\
4\end{array}$ \\
\hline Solanaceae & Nicotiana paniculata L. & $\begin{array}{l}\text { Kolish qora, } \\
\text { tabaco } \\
\text { cimarrón }\end{array}$ & & & & & & & & 1 & & 1 & $\begin{array}{r}0,0 \\
0\end{array}$ \\
\hline Solanaceae & Physalis peruviana $\mathrm{L}$. & Uvillas & 10 & 2 & & 22 & & 17 & & & & 51 & $\begin{array}{r}0,2 \\
5\end{array}$ \\
\hline Solanaceae & Salpichroa tristis Miers & $\begin{array}{l}\text { Shupllak, } \\
\text { shupllaku }\end{array}$ & 10 & 4 & & 20 & & 10 & & & & 44 & $\begin{array}{r}0,2 \\
2\end{array}$ \\
\hline Solanaceae & $\begin{array}{l}\text { Solanum habrochaites S. Knapp \& D.M. } \\
\text { Spooner }\end{array}$ & $\begin{array}{l}\text { Tunatomate, } \\
\text { asiaq tomate, } \\
\text { tomate qura }\end{array}$ & & 6 & & & & & & & & 6 & $\begin{array}{r}0,0 \\
3\end{array}$ \\
\hline Solanaceae & Solanum juninense Bitter & $\begin{array}{l}\text { Kashausu } \\
\text { macho }\end{array}$ & 1 & & & & & & & & & 1 & $\begin{array}{r}0,0 \\
0\end{array}$ \\
\hline Solanaceae & $\begin{array}{l}\text { Solanum nigrescens } \mathrm{M} . \text { Martens \& } \\
\text { Galeotti }\end{array}$ & $\begin{array}{l}\text { Qachuusu, } \\
\text { allqupaumllan }\end{array}$ & 20 & 5 & & & & 1 & & & & 26 & $\begin{array}{r}0,1 \\
3\end{array}$ \\
\hline Solanaceae & Solanum peruvianum L. & $\begin{array}{l}\text { Tunatomate, } \\
\text { asiaq tomate, } \\
\text { tomate qura }\end{array}$ & & 4 & & & & & & & & 4 & $\begin{array}{r}0,0 \\
2\end{array}$ \\
\hline $\begin{array}{l}\text { Thelypterid } \\
\text { aceae }\end{array}$ & $\begin{array}{l}\text { Thelypteris glandulosolanosa (C. Chr.) } \\
\text { R.M. Tryon }\end{array}$ & $\begin{array}{l}\text { Palma qora, } \\
\text { palma palma, } \\
\text { shishu }\end{array}$ & 10 & & & & & & 7 & & & 17 & $\begin{array}{r}0,0 \\
8\end{array}$ \\
\hline $\begin{array}{l}\text { Thelypterid } \\
\text { aceae }\end{array}$ & Thelypteris rufa (Poir.) A.R. Sm. & Palmera & 1 & & & & & & & & & 1 & $\begin{array}{r}0,0 \\
0\end{array}$ \\
\hline $\begin{array}{l}\text { Tropaeolac } \\
\text { eae }\end{array}$ & Tropaeolum minus L. & $\begin{array}{l}\text { Patuu, patee, } \\
\text { tuna mashua }\end{array}$ & 5 & 3 & & 13 & & & & 2 & & 23 & $\begin{array}{r}0,1 \\
1\end{array}$ \\
\hline Urticaceae & Phenax hirtus (Sw.) Wedd. & $\begin{array}{l}\text { Pukatullu, } \\
\text { pukaqeru }\end{array}$ & & 8 & 4 & & 5 & & & & & 17 & $\begin{array}{r}0,0 \\
8\end{array}$ \\
\hline Urticaceae & Urtica echinata Benth. & $\begin{array}{l}\text { Shishu shinua, } \\
\text { urqu shinua }\end{array}$ & 14 & & & & & & & & & 14 & $\begin{array}{r}0,0 \\
7\end{array}$ \\
\hline Urticaceae & Urtica flabellata Kunth & Ortiga china & 9 & & & & & & & & & 9 & $\begin{array}{r}0,0 \\
4\end{array}$ \\
\hline Urticaceae & Urtica leptophylla Kunth & $\begin{array}{l}\text { Shinua, ortiga } \\
\text { roja }\end{array}$ & 13 & 3 & & & & 1 & & & & 17 & $\begin{array}{r}0,0 \\
8\end{array}$ \\
\hline Urticaceae & Urtica urens L. & $\begin{array}{l}\text { Yanashinua, } \\
\text { ortiga negra }\end{array}$ & 8 & 1 & & & & & & & & 9 & $\begin{array}{r}0,0 \\
4\end{array}$ \\
\hline $\begin{array}{l}\text { Verbenacea } \\
\text { e }\end{array}$ & Citharexylum dentatum D.Don & Yana keru & & & 6 & & 2 & & & & & 8 & $\begin{array}{r}0,0 \\
4\end{array}$ \\
\hline
\end{tabular}


Apéndice 2. Lista de la flora silvestre útil del distrito de Pamparomás, Ancash. MED = Medicinal, APA = Alimento para animales, $\mathrm{COM}=$ Combustible, $\mathrm{ALI}=$ Alimenticio, $\mathrm{MAT}=$ Materiales, $\mathrm{SOC}=$ Social, $\mathrm{AMB}=$ Ambiental, $\mathrm{TOX}=$ Tóxica, ETN = Etnoveterinario, RU = Reportes de uso, IC = Importancia Cultural.

\begin{tabular}{|c|c|c|c|c|c|c|c|c|c|c|c|c|c|}
\hline Familia & Especie & $\begin{array}{l}\text { Nombre } \\
\text { vernáculo }\end{array}$ & $\begin{array}{l}\mathrm{M} \\
\mathrm{E} \\
\mathrm{D}\end{array}$ & $\begin{array}{l}\text { AP } \\
\text { A }\end{array}$ & $\begin{array}{l}\mathrm{CO} \\
\mathrm{M}\end{array}$ & $\begin{array}{l}\text { A } \\
\text { LI }\end{array}$ & $\begin{array}{l}\text { M } \\
\text { AT }\end{array}$ & $\begin{array}{l}\mathrm{SO} \\
\mathrm{C}\end{array}$ & $\begin{array}{l}\mathrm{AM} \\
\mathrm{B}\end{array}$ & $\begin{array}{l}\text { TO } \\
X\end{array}$ & $\begin{array}{l}\text { ET } \\
N\end{array}$ & RU & IC \\
\hline $\begin{array}{l}\text { Verbenacea } \\
\text { e }\end{array}$ & $\begin{array}{l}\text { Junellia fasciculata (Benth.) N.O'Leary \& } \\
\text { P.Peralta }\end{array}$ & $\begin{array}{l}\text { Azularia, } \\
\text { rankeneria }\end{array}$ & & & 10 & & & & 2 & & & 12 & $\begin{array}{r}0,0 \\
6\end{array}$ \\
\hline $\begin{array}{l}\text { Verbenacea } \\
\text { e }\end{array}$ & Verbena cuneifolia Ruiz \& Pav. & Azularia & & 4 & 4 & & & & & & & 8 & $\begin{array}{r}0,0 \\
4\end{array}$ \\
\hline Verbenacea & Verbena hispida Ruiz \& Pav. & $\begin{array}{l}\text { Verbena } \\
\text { silvestre }\end{array}$ & 15 & 4 & & & 3 & 3 & & 5 & & 30 & $\begin{array}{r}0,1 \\
5\end{array}$ \\
\hline $\begin{array}{l}\text { Verbenacea } \\
\text { e }\end{array}$ & Verbena pogostoma Klotzsch ex Walp. & Latak qura & & 4 & & & & & & & & 4 & $\begin{array}{r}0,0 \\
2\end{array}$ \\
\hline 68 & 237 & 234 & $\begin{array}{l}15 \\
87\end{array}$ & $\begin{array}{r}78 \\
6 \\
\end{array}$ & 595 & $\begin{array}{r}57 \\
2\end{array}$ & 389 & $\begin{array}{r}18 \\
8\end{array}$ & 133 & 43 & 55 & $\begin{array}{l}43 \\
48\end{array}$ & \\
\hline
\end{tabular}

${ }^{1}$ Departamento de Etnobotánica y Botánica Económica, Museo de Historia Natural, Universidad Nacional Mayor de San Marcos, Av. Arenales 1256, Jesús María, Lima, Perú.

${ }^{2}$ Facultad de Ciencias Veterinarias y Biológicas, Universidad Científica del Sur, Av. Antigua Carretera Panamericana Sur Km. 19, Villa El Salvador, Lima, Perú. Autor para correspondencia: e-mail Roxana Castañeda: castanedaroxana@gmail.com.

${ }^{3}$ Facultad de Ciencias Naturales y Matemática, Universidad Nacional Federico Villarreal, Jr. Río Chepén 290, El Agustino, Lima, Perú. 